\title{
NNLO QCD corrections to production of a spin-2 particle with nonuniversal couplings in the Drell-Yan process
}

\author{
Pulak Banerjee, ${ }^{1,2, *}$ Prasanna K. Dhani, ${ }^{1,2, \dagger}$ M. C. Kumar, ${ }^{3, \ddagger}$ Prakash Mathews, ${ }^{4,2, \S}$ and V. Ravindran ${ }^{1,2, \|}$ \\ ${ }^{1}$ The Institute of Mathematical Sciences, \\ IV Cross Road, CIT Campus, Chennai 600 113, Tamil Nadu, India \\ ${ }^{2}$ Homi Bhabha National Institute, Training School Complex, Anushakti Nagar, Mumbai 400085, India \\ ${ }^{3}$ Department of Physics, Indian Institute of Technology Guwahati, Guwahati 781039, India \\ ${ }^{4}$ Saha Institute of Nuclear Physics, I/AF Bidhan Nagar, Kolkata 700 064, West Bengal, India
}

(Received 7 November 2017; published 29 May 2018)

\begin{abstract}
We study the phenomenological impact of the interaction of spin-2 fields with those of the Standard Model in a model independent framework up to next-to-next-to-leading order in perturbative quantum chromodynamics. We use the invariant mass distribution of the pair of leptons produced at the Large Hadron Collider to demonstrate this. A minimal scenario where the spin-2 fields couple to two gauge invariant operators with different coupling strengths has been considered. These operators not being conserved show very different ultraviolet behavior increasing the searches options of spin-2 particles at the colliders. We find that our results using the higher order quantum corrections stabilize the predictions with respect to renormalization and factorization scales. We also find that corrections are appreciable which need to be taken into account in such searches at the colliders.
\end{abstract}

DOI: 10.1103/PhysRevD.97.094028

\section{INTRODUCTION}

With the absence of any signal of new physics at the Large Hadron Collider (LHC) at present energies, searches of physics beyond the Standard Model (BSM) are based on the ability to make very precise theoretical predictions within the Standard Model (SM) and to look for possible deviations between experimental observations and theoretical predictions, as a hint of new physics, within estimated uncertainties. In order to constrain the new physics model parameters, one needs to also compute the BSM signals to the same level of theoretical precision as the SM and compare with the observations made at the LHC. Quantum chromodynamics (QCD) corrections are large at the LHC and the inclusion of higher order terms reduces the theoretical uncertainties substantially. Many SM processes have been measured at the LHC and have cross sections that are in excellent agreement with higher order QCD predictions. This has helped in the discovery of the Higgs boson by

\footnotetext{
*bpulak@imsc.res.in

†prasannakd@imsc.res.in

*mckumar@iitg.ac.in

\$prakash.mathews@saha.ac.in

ravindra@imsc.res.in
}

Published by the American Physical Society under the terms of the Creative Commons Attribution 4.0 International license. Further distribution of this work must maintain attribution to the author(s) and the published article's title, journal citation, and DOI. Funded by SCOAP ${ }^{3}$.
ATLAS [1] and CMS [2] Collaborations at the LHC and hence the measurement of the important fundamental parameter of the SM, the Higgs mass $m_{H}$ (see [3-5]). Precise measurement of the Higgs mass is essential for the understanding of the stability of electroweak vacuum [6].

In spite of the fact that the SM is in excellent agreement with experimental observations, we know that there are compelling reasons to go beyond the SM. In the context of the discovery of a boson at $125 \mathrm{GeV}$ in the diphoton channel, models with spin-2 were also necessary to ascertain the spin and parity of the discovered boson. In the meanwhile the bounds on conventional models such as the Randall-Sundrum models with warped extra dimensions [7], where the spin-2 couples universally to the SM energy momentum tensor was much higher. A universally coupled spin-2 particle is heavily constrained [8,9]. Models with nonuniversal coupling of a spin-2 to SM was hence a suitable alternative. In this model, the spin- 2 couples to two sets of gauge invariant SM tensorial operators with different coupling strengths, but are not individually conserved. The universal coupling would correspond to the coupling strength being equal and the tensorial operators adding up to the conserved energy momentum tensor. Models with nonuniversal coupling were incorporated in tools such as Higgs characterization [10] to next-to-leading order (NLO) in QCD. Nonuniversal coupling leads to additional challenges: (a) additional UV renormalizations were needed; (b) in the IR sector, additional double and single pole terms had to be canceled with the counterparts from the real 
emission process and the mass factorization counterterms, thus demonstrating the IR factorization to NLO for nonuniversal coupling [10]. Note that we take this for granted in perturbative QCD and for universal coupling it is guaranteed by the conserved energy-momentum tensor.

Recently, the UV structure of nonuniversal coupling up to three-loop order in QCD was investigated [11] where in the spin-2 fields couple to two sets of gauge invariant tensorial operators constructed out of the SM fields (with different coupling strengths). These rank-2 operators are unfortunately not conserved, unlike the energy-momentum tensor of QCD [12]. Consequently, both these operators as well as the couplings get additional UV renormalization order by order in perturbation theory. Exploiting the universal IR structure of QCD amplitudes even in the case of a nonuniversal spin-2 coupling, on-shell form factors of these operators between quark and gluon states have been computed. These are important ingredients for observables at the LHC, to study models with such interactions.

For universal coupling, depending on the geometry of extra dimensions, viz. large extra dimensions or warped extra dimension models, studies have been extensively carried out up to higher orders in QCD in various channels that are relevant for the LHC. In these models, the Drell-Yan (DY) process has been studied to NLO [13-15] for various observables. Divector boson final states have been studied to the NLO level in [16-21]. To NLO + parton shower(PS) accuracy all the noncolor, difinal states have been studied [22-24] in the aMC@NLO framework. Production of a generic spin-2 particle in association with colored particles, vector bosons, and the Higgs boson have been studied in [25] to NLO + PS accuracy. To the next higher order in QCD the form factor of a spin-2 universally coupled to quarks and gluons up to two loops was computed in [26]. Subsequently the next-to-next-to-leading order (NNLO) computation in the threshold limit was done in [27] and finally the full NNLO computation in [28]. Production of a spin-2 in association with a jet to full two-loop QCD corrections has also been completed recently with the evaluation of generic spin-2 decaying to $g g g$ [29] and $q \bar{q} g$ [30].

The dilepton final state is the most studied and a very clean process at the LHC. In BSM scenarios the dilepton signal could be enhanced due to additional contributions from BSM intermediate states that could couple to a dilepton. For the universal spin- 2 coupling the DY process has been evaluated up to NNLO in QCD. This involved various steps: to begin with NLO corrections were evaluated [13], followed by the two-loop quark and gluon form factors [26], which lead to the computation of NNLO QCD corrections to the graviton production in models of $\mathrm{TeV}$-scale gravity, within the softvirtual approximation [27]. Finally the complete NNLO QCD corrections to the production of dileptons at hadron colliders in large extra dimension models with spin-2 particles are reported in [28].
The nonuniversal coupling of spin-2 to SM has been actively considered by the ATLAS Collaboration [31,32] to provide exclusion of several non-SM spin hypotheses. This analysis has been done in the Higgs characterization framework $[10,25]$ to NLO + PS accuracy. With the recent results [11] up to three-loop form factors of a massive spin-2 particle with nonuniversal coupling, NNLO computation is now possible. In this article we look at the phenomenological implications of these models to NNLO at the LHC.

The paper is organized as follows. We discuss the effective action that describes how a spin-2 particle couples to those of the SM through two gauge invariant operators with renormalizable coupling. Using this action, we compute QCD radiative correction to the production of a pair of leptons, in particular their invariant mass distribution up to the NNLO level. A detailed phenomenological study on the impact of our results is presented for the LHC. Finally we conclude. The relevant form factors are presented in the Appendix, and mass factorized partonic cross sections are given as an electronically readable version.

\section{THEORETICAL FRAMEWORK}

\section{A. Effective action}

The interaction part of the effective action describes the nonuniversal coupling of the spin-2 fields denoted by $h_{\mu \nu}$ with those of QCD, consists of two gauge invariant operators, namely $\hat{\mathcal{O}}_{\mu \nu}^{G}$ and $\hat{\mathcal{O}}_{\mu \nu}^{Q}$, and is given by

$$
S=-\frac{1}{2} \int d^{4} x h^{\mu \nu}(x)\left(\hat{\kappa}_{G} \hat{\mathcal{O}}_{\mu \nu}^{G}(x)+\hat{\kappa}_{Q} \hat{\mathcal{O}}_{\mu \nu}^{Q}(x)\right),
$$

where $\hat{\kappa}_{G, Q}$ are dimension full couplings, the pure gauge sector is denoted by $G$, while $Q$ denotes the fermionic sector and its gauge interaction. This decomposition is not unique as one can adjust gauge invariant terms between them. The gauge invariant operators $\hat{\mathcal{O}}_{\mu \nu}^{G}$ and $\hat{\mathcal{O}}_{\mu \nu}^{Q}$ are as follows:

$$
\begin{aligned}
\hat{\mathcal{O}}_{\mu \nu}^{G}= & \frac{1}{4} g_{\mu \nu} \hat{F}_{\alpha \beta}^{a} \hat{F}^{a \alpha \beta}-\hat{F}_{\mu \rho}^{a} \hat{F}_{\nu}^{a \rho}-\frac{1}{\hat{\xi}} g_{\mu \nu} \partial^{\rho}\left(\hat{A}_{\rho}^{a} \partial^{\sigma} \hat{A}_{\sigma}^{a}\right) \\
& -\frac{1}{2 \hat{\xi}} g_{\mu \nu} \partial_{\alpha} \hat{A}^{a \alpha} \partial_{\beta} \hat{A}^{a \beta}+\frac{1}{\hat{\xi}}\left(\hat{A}_{\nu}^{a} \partial_{\mu}\left(\partial^{\sigma} \hat{A}_{\sigma}^{a}\right)+\hat{A}_{\mu}^{a} \partial_{\nu}\left(\partial^{\sigma} \hat{A}_{\sigma}^{a}\right)\right) \\
& +\partial_{\mu} \overline{\hat{\omega}}^{a}\left(\partial_{\nu} \hat{\omega}^{a}-\hat{g}_{s} f^{a b c} \hat{A}_{\nu}^{c} \hat{\omega}^{b}\right) \\
& +\partial_{\nu} \hat{\hat{\omega}}^{a}\left(\partial_{\mu} \hat{\omega}^{a}-\hat{g}_{s} f^{a b c} \hat{A}_{\mu}^{c} \hat{\omega}^{b}\right) \\
& -g_{\mu \nu} \partial_{\alpha} \overline{\hat{\omega}^{a}}\left(\partial^{\alpha} \hat{\omega}^{a}-\hat{g}_{s} f^{a b c} \hat{A}^{c \alpha} \hat{\omega}^{b}\right), \\
\hat{\mathcal{O}}_{\mu \nu}^{Q}= & \frac{i}{4}\left[\overline{\hat{\psi}} \gamma_{\mu}\left(\vec{\partial}_{\nu}-i \hat{g}_{s} T^{a} \hat{A}_{\nu}^{a}\right) \hat{\psi}-\overline{\hat{\psi}}\left(\overleftarrow{\partial}_{\nu}+i \hat{g}_{s} T^{a} \hat{A}_{\nu}^{a}\right) \gamma_{\mu} \hat{\psi}\right. \\
& \left.+\overline{\hat{\psi}} \gamma_{\nu}\left(\vec{\partial}_{\mu}-i \hat{g}_{s} T^{a} \hat{A}_{\mu}^{a}\right) \hat{\psi}-\overline{\hat{\psi}}\left(\grave{\partial}_{\mu}+i \hat{g}_{s} T^{a} \hat{A}_{\mu}^{a}\right) \gamma_{\nu} \hat{\psi}\right] \\
& -i g_{\mu \nu} \overline{\hat{\psi}} \gamma^{\alpha}\left(\vec{\partial}_{\alpha}-i \hat{g}_{s} T^{a} \hat{A}_{\alpha}^{a}\right) \hat{\psi},
\end{aligned}
$$

and in the above equations the unrenormalized quantities are denoted by a hat $(\hat{)}) \cdot \hat{g}_{s}$ is the strong coupling constant, $\hat{\xi}$ the 
gauge fixing parameter, $\hat{A}_{\nu}^{c}$ the gauge field, $\hat{\psi}$ the quark field, and $\hat{\omega}^{a}$ the ghost fields. The structure constants of the $S U(N)$ gauge group are denoted by $f^{a b c}$ and the Gell-Mann matrices by $T^{a}$. The sum of $\hat{\mathcal{O}}_{G}$ and $\hat{\mathcal{O}}_{Q}$ is the energy momentum tensor of the QCD part and is protected by radiative corrections to all orders, thanks to the fact that it is conserved. The Feynman rules for the nonuniversal case in contrast to the universal case $[33,34]$ would have a prefactor $\kappa_{Q}$ for the coupling for a spin-2 to a pair of fermions or any fermionic SM vertex, while a spin-2 coupling to gluons, ghosts, or any SM gauge or ghost vertex would have a prefactor $\kappa_{G}$. The individual gauge $\mathcal{O}_{G}$ and fermionic $\mathcal{O}_{Q}$ operators are not conserved in $\mathrm{QCD}$ and hence require additional ultraviolet (UV) counterterms in order to renormalize them. In [11], we determined these additional UV renormalization constants up to the three-loop level in QCD. We obtained them by exploiting the universal infrared properties of on-shell amplitudes involving these composite operators. Since we have two operators at our disposal, they mix under renormalization as follows:

$$
\left[\begin{array}{c}
O^{G} \\
O^{Q}
\end{array}\right]=\left[\begin{array}{ll}
Z_{G G} & Z_{G Q} \\
Z_{Q G} & Z_{Q Q}
\end{array}\right]\left[\begin{array}{c}
\hat{O}^{G} \\
\hat{O}^{Q}
\end{array}\right],
$$

where the renormalization constants $Z_{I J}$ in terms of the anomalous dimensions $\gamma_{I J}=\sum_{n=1}^{\infty} a_{s}^{n} \gamma_{I J}^{(n)}$ are given by

$$
\begin{aligned}
Z_{I J}= & \delta_{I J}+a_{s}\left[\frac{2}{\epsilon} \gamma_{I J}^{(1)}\right] \\
& +a_{s}^{2}\left[\frac{1}{\epsilon^{2}}\left\{2 \beta_{0} \gamma_{I J}^{(1)}+2 \gamma_{I K}^{(1)} \gamma_{K J}^{(1)}\right\}+\frac{1}{\epsilon}\left\{\gamma_{I J}^{(2)}\right\}\right],
\end{aligned}
$$

where $I, J=G, Q, a_{s} \equiv g_{s}^{2} / 16 \pi^{2}$, and the space-time dimension is taken to be $d=4+\epsilon$. The renormalization constants $Z_{I J}$ computed in [11] are given below up to $a_{s}^{2}$ for completeness:

$$
\begin{aligned}
& Z_{G G}=1+a_{s}\left[-\frac{4}{3 \epsilon} n_{f}\right]+a_{s}^{2}\left[\frac{1}{\epsilon^{2}}\left\{-\frac{44}{9} C_{A} n_{f}+\frac{32}{9} C_{F} n_{f}+\frac{16}{9} n_{f}^{2}\right\}+\frac{1}{\epsilon}\left\{-\frac{35}{27} C_{A} n_{f}-\frac{74}{27} C_{F} n_{f}\right\}\right], \\
& Z_{G Q}=a_{s}\left[\frac{16}{3 \epsilon} C_{F}\right]+a_{s}^{2}\left[\frac{1}{\epsilon^{2}}\left\{\frac{176}{9} C_{A} C_{F}-\frac{64}{9} C_{F} n_{f}-\frac{128}{9} C_{F}^{2}\right\}+\frac{1}{\epsilon}\left\{\frac{376}{27} C_{A} C_{F}-\frac{104}{27} C_{F} n_{f}-\frac{112}{27} C_{F}^{2}\right\}\right], \\
& Z_{Q G}=a_{s}\left[\frac{4}{3 \epsilon} n_{f}\right]+a_{s}^{2}\left[\frac{1}{\epsilon^{2}}\left\{\frac{44}{9} C_{A} n_{f}-\frac{32}{9} C_{F} n_{f}-\frac{16}{9} n_{f}^{2}\right\}+\frac{1}{\epsilon}\left\{\frac{35}{27} C_{A} n_{f}+\frac{74}{27} C_{F} n_{f}\right\}\right], \\
& Z_{Q Q}=1+a_{s}\left[-\frac{16}{3 \epsilon}\right]+a_{s}^{2}\left[\frac{1}{\epsilon^{2}}\left\{-\frac{176}{9} C_{A} C_{F}+\frac{64}{9} C_{F} n_{f}+\frac{128}{9} C_{F}^{2}\right\}+\frac{1}{\epsilon}\left\{-\frac{376}{27} C_{A} C_{F}+\frac{104}{27} C_{F} n_{f}+\frac{112}{27} C_{F}^{2}\right\}\right],
\end{aligned}
$$

where $C_{A}=N$ and $C_{F}=\left(N^{2}-1\right) / 2 N$ are the quadratic Casimirs of the $S U(N)$ group and $n_{f}$ is the number of quark flavors. The fact that the energy momentum tensor $T_{\mu \nu}=$ $\mathcal{O}_{\mu \nu}^{G}+\mathcal{O}_{\mu \nu}^{Q}$ is conserved leads to $\gamma_{Q G}^{(n)}=-\gamma_{G G}^{(n)}$ and $\gamma_{Q Q}^{(n)}=$ $-\gamma_{G Q}^{(n)}$ or equivalently $Z_{G G}=1-Z_{Q G}$ and $Z_{Q Q}=1-Z_{G Q}$, which is expected to be true to all orders in $a_{s}$. All $\gamma_{G G}^{(n)}$ are proportional to $n_{f}$ which is consistent with the expectation that the conserved property of $\mathcal{O}_{\mu \nu}^{G}$ breaks down beyond tree level due to the presence of quark loops. For pure gauge theory $\left(n_{f}=0\right)$ and the energy momentum tensor of the pure gauge theory $\mathcal{O}_{\mu \nu}^{G}$ is hence conserved by itself.

Defining the renormalized $\kappa_{I}$ in terms of bare ones through $\hat{\kappa}_{I}=\sum_{J=G, Q} Z_{I J} \kappa_{J}$ with $I, J=G, Q$, we find that the action takes the following form:

$$
S=-\frac{1}{2} \int d^{4} x h_{\mu \nu}\left(\kappa_{G} \mathcal{O}^{G, \mu \nu}+\kappa_{Q} \mathcal{O}^{Q, \mu \nu}\right)
$$

the resulting interaction terms expressed in terms of renormalized operators and renormalized couplings are guaranteed to predict UV finite quantities to all orders in strong coupling. In the rest of the paper, we will use this version of the Lagrangian to study the phenomenology.

\section{B. Lepton pair invariant mass distribution $d \sigma / d Q^{2}$}

Our next task is to use the effective action expressed in terms of renormalized operators $\mathcal{O}_{I}$ and couplings $\kappa_{I}$ to obtain the production cross section for a pair of leptons $\left(l^{+}, l^{-}\right)$, through the scattering of two protons $H_{1}, H_{2}$ at the LHC,

$$
H_{1}\left(P_{1}\right)+H_{2}\left(P_{2}\right) \rightarrow l^{+}\left(l_{1}\right)+l^{-}\left(l_{2}\right)+X\left(P_{X}\right),
$$

where the 4-momenta of the corresponding particles are denoted in the parentheses and the final inclusive state is denoted by $X$. The hadronic cross section is related to the partonic subprocess cross sections in the QCD improved parton model as

$$
\begin{aligned}
2 S \frac{d \sigma^{H_{1} H_{2}}}{d Q^{2}}\left(\tau, Q^{2}\right)= & \sum_{a b=q, \bar{q}, g} \int_{0}^{1} d x_{1} \int_{0}^{1} d x_{2} \hat{f}_{a}^{H_{1}}\left(x_{1}\right) \hat{f}_{b}^{H_{2}}\left(x_{2}\right) \\
& \times \int_{0}^{1} d z 2 s \frac{d \hat{\sigma}^{a b}}{d Q^{2}}\left(z, Q^{2}\right) \delta\left(\tau-z x_{1} x_{2}\right),
\end{aligned}
$$


where $Q^{2}$ is the invariant mass square of the final state leptonic pair and $S$ is the square of the hadronic center of mass energy which is related to the partonic one, $s$, through $s=x_{1} x_{2} S$, and similarly $\tau \equiv Q^{2} / S, z \equiv Q^{2} / s$, and $\tau=x_{1} x_{2} z$. The unrenormalized partonic distribution functions of the partons $a$ and $b$ are $\hat{f}_{a}$ and $\hat{f}_{b}$, respectively. The partonic subprocess corresponding to the hadronic process is

$$
a\left(p_{1}\right)+b\left(p_{2}\right) \rightarrow j(q)+\sum_{i=1}^{m} X_{i}\left(q_{i}\right),
$$

where the summation over $i$ corresponds to all the real QCD final state partons that could contribute to a particular order in perturbative QCD. The initial state partons $a b \rightarrow j$, a neutral state $j$ which could be a photon $\left(\gamma^{*}\right), Z$-boson $\left(Z^{*}\right)$, or spin-2 particle and further decays to a pair of leptons $j \rightarrow l^{+} l^{-}$.

At the partonic level, one encounters amplitudes involving both SM vector bosons and spin-2 particles as propagators, and hence, at the cross section level, the squared amplitudes contain in addition to contributions from SM and spin-2 separately those from interference of SM and spin-2 amplitudes. Interestingly, for the invariant mass distributions, the later one identically vanishes for the universal case, which was earlier noted at both NLO and NNLO levels in $[13,28]$. Hence, at the cross section level, the SM and spin-2 contributions simply add up as

$2 S \frac{d \sigma^{H_{1} H_{2}}}{d Q^{2}}\left(\tau, Q^{2}\right)=2 S \frac{d \sigma_{\mathrm{SM}}^{H_{1} H_{2}}}{d Q^{2}}\left(\tau, Q^{2}\right)+2 S \frac{d \sigma_{\mathrm{spin}-2}^{H_{1} H_{2}}}{d Q^{2}}\left(\tau, Q^{2}\right)$,

where the SM results are known exactly up to the NNLO level for a long time (see [35-38]) and the result at $\mathrm{N}^{3} \mathrm{LO}$ in the soft gluon approximation is also available; see [39]. For the spin-2 case with universal coupling, namely $\kappa_{G}=\kappa_{Q}=\kappa$, the results up to the NNLO level can be found in $[13,28]$. In this article, we have extended this computation to NNLO QCD for the case of nonuniversal couplings, i.e., when $\kappa_{G}$ and $\kappa_{Q}$ are different. We briefly describe the methodology that we use to obtain the mass factorized partonic cross sections up to the NNLO level. Unlike the SM, for the spin-2 exchange, at leading order (LO) we can have a gluon initiated subprocess in addition to the quark initiated one:

$$
q+\bar{q} \rightarrow l^{+} l^{-}, \quad g+g \rightarrow l^{+} l^{-} .
$$

At NLO in QCD, we have

$$
\begin{aligned}
q+\bar{q} & \rightarrow l^{+} l^{-}+g, & q+\bar{q} & \rightarrow l^{+} l^{-}+\text {one loop, } \\
g+g & \rightarrow l^{+} l^{-}+g, & g+g & \rightarrow l^{+} l^{-}+\text {one loop, } \\
g+q & \rightarrow l^{+} l^{-}+q, & g+\bar{q} & \rightarrow l^{+} l^{-}+\bar{q} .
\end{aligned}
$$

At NNLO level, we have double real emission,

$$
\begin{array}{ll}
q+\bar{q} \rightarrow l^{+} l^{-}+q+\bar{q}, & q_{1}+\bar{q}_{2} \rightarrow l^{+} l^{-}+q_{1}+\bar{q}_{2}, \\
g+g \rightarrow l^{+} l^{-}+g+g, & q_{1}+\bar{q}_{1} \rightarrow l^{+} l^{-}+q_{2}+\bar{q}_{2}, \\
g+q \rightarrow l^{+} l^{-}+g+q, & q+\bar{q} \rightarrow l^{+} l^{-}+g+g, \\
q+q \rightarrow l^{+} l^{-}+q+q, & g+g \rightarrow l^{+} l^{-}+q+\bar{q}, \\
g+\bar{q} \rightarrow l^{+} l^{-}+g+\bar{q}, & q_{1}+q_{2} \rightarrow l^{+} l^{-}+q_{1}+q_{2},
\end{array}
$$

single real emission at one loop,

$$
\begin{aligned}
& q+\bar{q} \rightarrow l^{+} l^{-}+g+\text { one loop, } \\
& g+g \rightarrow l^{+} l^{-}+g+\text { one loop, } \\
& g+q \rightarrow l^{+} l^{-}+q+\text { one loop, } \\
& g+\bar{q} \rightarrow l^{+} l^{-}+\bar{q}+\text { one loop, }
\end{aligned}
$$

and the pure double virtual diagrams,

$$
\begin{aligned}
q+\bar{q} & \rightarrow l^{+} l^{-}+\text {two loop, } \\
g+g & \rightarrow l^{+} l^{-}+\text {two loop. }
\end{aligned}
$$

The virtual corrections at one- and two-loop levels are straightforward for this process, and the phase space integrals are often hard to evaluate. In the first computation of the NNLO QCD correction to the DY pair production [38], the phase space integrals were performed in three different frames to achieve the final result. This method was successfully applied in [5] to obtain inclusive cross section for the Higgs production at NNLO. In [3], using a systematic expansion around threshold, all the phase space integrals were performed to obtain the partonic cross sections for both DY and Higgs productions at the NNLO level. Later on, in [4], an elegant formalism was developed to compute both real emissions as well as virtual corrections applying integration by parts [40,41] and Lorentz invariance [42] identities. This approach is famously called the method of reverse unitarity. The resulting master integrals were computed using the technique of differential equations. The stateof-the-art result, namely, the $\mathrm{N}^{3} \mathrm{LO}$ QCD corrections to the inclusive Higgs boson production [43-45], uses the method of reverse unitarity. We have systematically used this approach [4] to calculate the partonic cross section of the DY pair production through the intermediate spin-2 particle at NNLO QCD.

Ultraviolet, soft, and collinear (IR) divergences do show up beyond leading order, and they are regularized in dimensional regularization where the space-time dimensions $d$ are chosen to be equal to $4+\epsilon$. The soft divergences cancel among virtual and real subprocesses and processes thanks to the Kinoshita-Lee-Nauenberg theorem [46,47], and the remaining UV divergences as well as the initial state 
collinear divergences are removed in the M̄S scheme using UV renormalization constants and mass factorization kernels denoted by $\Gamma_{a b}\left(\mu_{F}\right)$, respectively. Here, $\mu_{F}$ is the factorization scale. For the UV renormalization, we need to perform renormalization for strong coupling constant $a_{s}=g_{s}^{2} / 16 \pi^{2}$ through $Z_{a_{s}}$ as well as renormalization of $\kappa_{I}$ through $Z_{I J}$ listed in the previous section. For the former, we have

$$
\hat{a}_{s} S_{\epsilon}=\left(\frac{\mu^{2}}{\mu_{R}^{2}}\right)^{\epsilon / 2} Z_{a_{s}} a_{s}
$$

where

$$
Z_{a_{s}}=1+a_{s}\left[\frac{2}{\epsilon} \beta_{0}\right]+a_{s}^{2}\left[\frac{4}{\epsilon^{2}} \beta_{0}^{2}+\frac{1}{\epsilon} \beta_{1}\right]+\cdots,
$$

$a_{s} \equiv a_{s}\left(\mu_{R}^{2}\right), \quad S_{\epsilon}=\exp \left[\left(\gamma_{E}-\ln 4 \pi\right) \epsilon / 2\right], \gamma_{E}=0.5772 \ldots$, and the scale $\mu$ is introduced to keep the unrenormalized strong coupling constant $\hat{a}_{s}$ dimensionless in $n$ dimensions. The renormalization scale is denoted by $\mu_{R}$. $\beta_{i}$ 's are the coefficients of the QCD $\beta$-function [48-52]. The mass factorized finite cross section can be obtained using

$$
\begin{aligned}
& 2 s \frac{d \hat{\sigma}_{a b}}{d Q^{2}}\left(z, Q^{2}, 1 / \epsilon\right) \\
& \quad=\sum_{c, d=q, \bar{q}, g} \Gamma_{c a}\left(z, \mu_{F}^{2}, 1 / \epsilon\right) \otimes \Gamma_{d b}\left(z, \mu_{F}^{2}, 1 / \epsilon\right) \\
& \quad \otimes 2 s \frac{d \sigma_{a b}}{d Q^{2}}\left(z, Q^{2}, \mu_{F}^{2}\right)
\end{aligned}
$$

where $\otimes$ are nothing but Mellin convolution. The mass factorization kernels take the following form:

$$
\begin{aligned}
\Gamma_{a b}\left(z, \mu_{F}^{2}, 1 / \epsilon\right)= & \delta_{a b} \delta(1-z)+a_{s}\left(\mu_{F}^{2}\right) \frac{1}{\epsilon} P_{a b}^{(0)}(z) \\
& +a_{s}^{2}\left(\mu_{F}^{2}\right)\left[\frac{1}{\epsilon^{2}}\left(\frac{1}{2} P_{a c}^{(0)} \otimes P_{c b}^{(0)}+\beta_{0} P_{a b}^{(0)}\right)\right. \\
& \left.+\frac{1}{\epsilon}\left(\frac{1}{2} P_{a b}^{(1)}\right)\right]+\cdots,
\end{aligned}
$$

where $P_{a b}^{(i)}$ are the Altarelli-Parisi splitting functions [53-58]. After the mass factorization, the finite partonic cross sections denoted by $2 s d \sigma_{a b} / d Q^{2}$ can be expressed in terms $\Delta_{a b}^{h}\left(z, a_{s}\left(\mu_{R}^{2}\right), Q^{2} / \mu_{R}^{2}, \mu_{F}^{2} / \mu_{R}^{2}\right)$ by factoring out some overall constants. In terms of these $\Delta_{a b}^{h}$, the hadronic cross section can be written as

$$
\begin{aligned}
2 S \frac{d \sigma_{\text {spin-2 }}^{H_{1} H_{2}}}{d Q^{2}}\left(\tau, Q^{2}\right)= & \sum_{q, \bar{q}, g} \mathcal{F}_{h} \int_{0}^{1} d x_{1} \int_{0}^{1} d x_{2} \int_{0}^{1} d z \delta\left(\tau-z x_{1} x_{2}\right) \times\left[H_{q \bar{q}} \sum_{k=0}^{2} a_{s}^{k} \Delta_{q \bar{q}}^{h,(k)}+H_{g g} \sum_{k=0}^{2} a_{s}^{k} \Delta_{g g}^{h,(k)}\right. \\
& \left.+\left(H_{g q}+H_{q g}\right) \sum_{k=1}^{2} a_{s}^{k} \Delta_{g q}^{h,(k)}+H_{q q} \sum_{k=2}^{2} a_{s}^{k} \Delta_{q q}^{h,(k)}+H_{q_{1} q_{2}} \sum_{k=2}^{2} a_{s}^{k} \Delta_{q_{1} q_{2}}^{h,(k)}\right]
\end{aligned}
$$

where

$$
\mathcal{F}_{h}=\frac{\kappa_{Q}^{2} Q^{6}}{320 \pi^{2}}\left|\mathcal{D}\left(Q^{2}\right)\right|^{2}, \quad \Delta_{a b}^{h,(k)}=\Delta_{a b}^{h,(k)}\left(z, \frac{Q^{2}}{\mu_{R}^{2}}, \frac{\mu_{F}^{2}}{\mu_{R}^{2}}\right) .
$$

$\kappa_{Q}$ in $\mathcal{F}_{h}$ corresponds to the leptonic coupling to the spin-2, while the couplings to quarks and gluons are taken in $\Delta_{a b}^{h,(k)}$. $\mathcal{D}\left(Q^{2}\right)$ is the propagator of the massive spin-2 particle, with a decay width that has to be estimated considering its decay to SM particles. $H_{a b}$ are the combinations of the mass factorized partonic distribution functions:

$$
\begin{aligned}
H_{q \bar{q}}\left(x_{1}, x_{2}, \mu_{F}^{2}\right) & =f_{q}^{H_{1}}\left(x_{1}, \mu_{F}^{2}\right) f_{\bar{q}}^{H_{2}}\left(x_{2}, \mu_{F}^{2}\right)+f_{\bar{q}}^{H_{1}}\left(x_{1}, \mu_{F}^{2}\right) f_{q}^{H_{2}}\left(x_{2}, \mu_{F}^{2}\right), \\
H_{q q}\left(x_{1}, x_{2}, \mu_{F}^{2}\right) & =f_{q}^{H_{1}}\left(x_{1}, \mu_{F}^{2}\right) f_{q}^{H_{2}}\left(x_{2}, \mu_{F}^{2}\right)+f_{\bar{q}}^{H_{1}}\left(x_{1}, \mu_{F}^{2}\right) f_{\bar{q}}^{H_{2}}\left(x_{2}, \mu_{F}^{2}\right), \\
H_{q_{1} q_{2}}\left(x_{1}, x_{2}, \mu_{F}^{2}\right) & =f_{q_{1}}^{H_{1}}\left(x_{1}, \mu_{F}^{2}\right)\left(f_{q_{2}}^{H_{2}}\left(x_{2}, \mu_{F}^{2}\right)+f_{\bar{q}_{2}}^{H_{2}}\left(x_{2}, \mu_{F}^{2}\right)\right)+f_{\bar{q}_{1}}^{H_{1}}\left(x_{1}, \mu_{F}^{2}\right)\left(f_{q_{2}}^{H_{2}}\left(x_{2}, \mu_{F}^{2}\right)+f_{\bar{q}_{2}}^{H_{2}}\left(x_{2}, \mu_{F}^{2}\right)\right), \\
H_{g q}\left(x_{1}, x_{2}, \mu_{F}^{2}\right) & =f_{g}^{H_{1}}\left(x_{1}, \mu_{F}^{2}\right)\left(f_{q}^{H_{2}}\left(x_{2}, \mu_{F}^{2}\right)+f_{\bar{q}}^{H_{2}}\left(x_{2}, \mu_{F}^{2}\right)\right), \\
H_{q g}\left(x_{1}, x_{2}, \mu_{F}^{2}\right) & =H_{g q}\left(x_{2}, x_{1}, \mu_{F}^{2}\right) \\
H_{g g}\left(x_{1}, x_{2}, \mu_{F}^{2}\right) & =f_{g}^{H_{1}}\left(x_{1}, \mu_{F}^{2}\right) f_{g}^{H_{2}}\left(x_{2}, \mu_{F}^{2}\right) .
\end{aligned}
$$

In the next section, we study the numerical implication of NNLO QCD corrections to a spin-2 coupling nonuniversally to the SM in the DY process. 


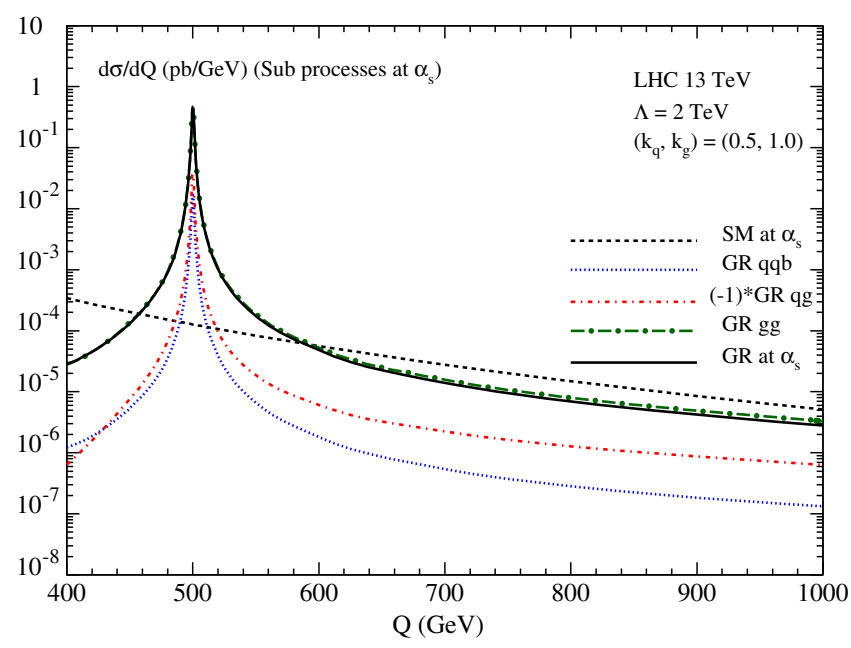

FIG. 1. First order QCD corrections from different subprocesses to dilepton production. The choice of the model parameters is as mentioned in the text.

\section{NUMERICAL RESULTS}

In this section, we present the numerical impact of our NNLO results on the production of dileptons at the LHC. We considered a minimal scenario of nonuniversal couplings of a spin-2 particle with SM fields, where the spin-2 particle couples to all SM fermions with coupling $\kappa_{Q}=$ $\sqrt{2} k_{q} / \Lambda$ and to all SM gauge bosons with a coupling strength of $\kappa_{G}=\sqrt{2} k_{g} / \Lambda$. Numerical results presented in this section are for the default choice of model parameters, namely, the spin- 2 particle of mass $m_{G}=500 \mathrm{GeV}$, the scale $\Lambda=2 \mathrm{TeV}$, and the couplings $\left(k_{q}, k_{g}\right)=(0.5,1.0)$. Both the renormalization and factorization scales are set equal to the invariant mass of the dilepton, i.e., $\mu_{R}=\mu_{F}=Q$. Throughout, we use MSTW2008nnlo parton distribution functions (PDFs) with the corresponding $a_{s}$

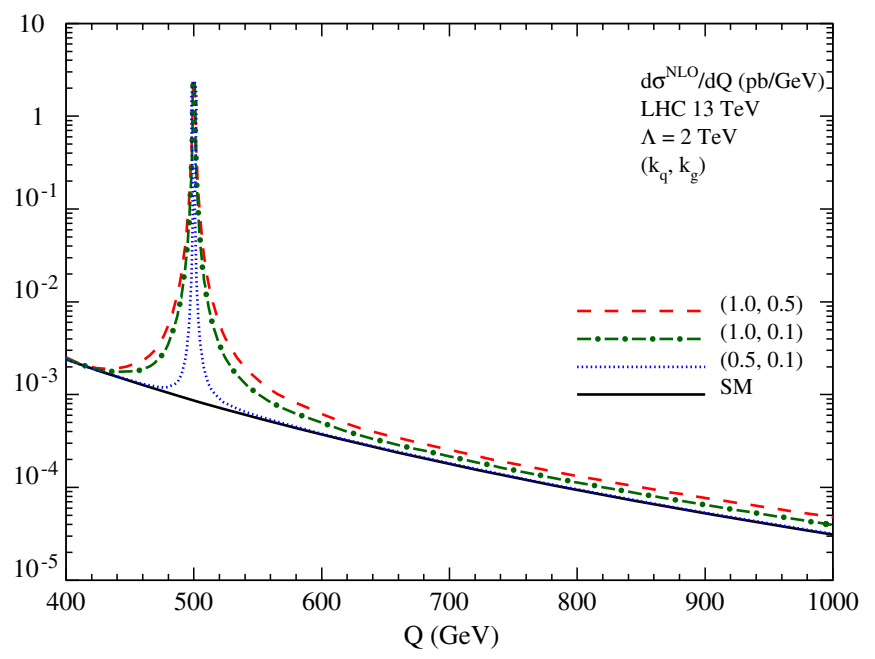

provided from LHAPDF unless otherwise stated. They chose $\sqrt{S}=13 \mathrm{TeV}$, the center of mass energy of the incoming hadrons at the LHC.

In our analysis, we restricted ourselves to the situation where the spin-2 particle decays only to SM fields. The spin-2 particle decay widths for nonuniversal couplings are the same as those given in [33]. For the scenario taken up here, where in all spin- 2 coupling to all bosons are taken to be identical, we note that the spin-2 particle decaying to $Z \gamma$ vanishes identically $\Gamma(h \rightarrow Z \gamma)=0$ [59]. In Fig. 1, we present the NLO corrections (only at order $a_{s}$ ) from various subprocess contributions to the dilepton production. For our default choice of model parameters, we find that the $g g$ subprocess contribution dominates over the rest. In general, the total NLO correction is smaller than the $g g$ contribution because of the negative contribution from the $q g$ subprocess. We also note that the $g g$ has a dominant contribution to the total decay width for couplings $(0.5,1.0)$.

To estimate the impact of QCD corrections, we define the K-factors as follows:

$$
\mathrm{K}_{1}=\frac{d \sigma^{\mathrm{NLO}} / d Q}{d \sigma^{\mathrm{LO}} / d Q} \quad \text { and } \quad \mathrm{K}_{2}=\frac{d \sigma^{\mathrm{NNLO}} / d Q}{d \sigma^{\mathrm{LO}} / d Q} .
$$

In the left panel of Fig. 2, we present dilepton invariant mass distributions to NLO for different choices of nonuniversal couplings $\left(k_{q}, k_{g}\right)=(1.0,0.5),(1.0,0.1)$, and $(0.5,0.1)$. It is expected for universal couplings that at the resonance region, the cross sections, i.e., the height of the peak, will be the same simply because the couplings at the matrix element level will cancel with those from the decay width of the spin-2 particle. However, for nonuniversal couplings this is not the case and hence cross sections at the resonance for different nonuniversal couplings will be different. Thus, the precision as well as the phenomenological studies of the spin-2 particle production

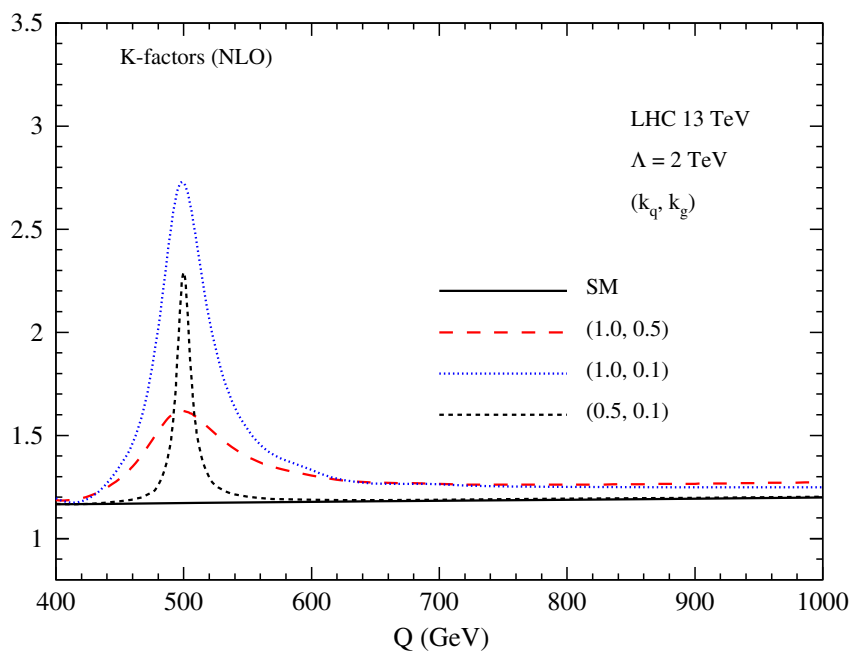

FIG. 2. Dilepton invariant mass distributions are presented to NLO QCD for different choices of couplings $\left(k_{q}\right.$, $\left.k_{g}\right)$ in the left panel. The corresponding K-factors are presented in the right panel. 


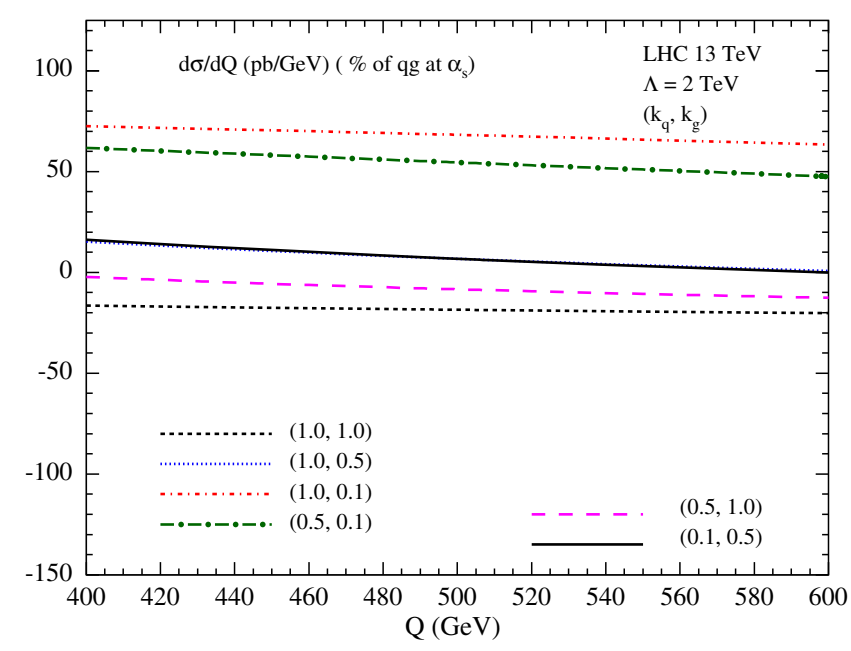

FIG. 3. Percentage of $q g$ subprocess contribution $R_{q g}^{(1)}$ as defined in the text for different choices of nonuniversal couplings.

in this model will be different from those of the warped extra dimension models. The NLO K-factor $\left(K_{1}\right)$ is present in the right panel for various choices of $\left(k_{q}, k_{g}\right)$, and we observe that the K-factor crucially depends on the choice of nonuniversal couplings. In particular we notice that the K-factors are larger for the choice of couplings $(1 \cdot 0,0.1)$. To understand this behavior better, it is helpful to study the percentage contribution of various subprocesses to the total correction at the NLO level, particularly from the $q g$ subprocess due to its large flux at LHC energies. In particular we define the percentage of the contribution of a given subprocess $a b$ as $R_{a b}^{(i)}=\left(d \sigma_{a b}^{H_{1} H_{2},(i)} / d Q^{2}\right) /$ $\left(d \sigma^{H_{1} H_{2},(i)} / d Q^{2}\right) \times 100$, where the numerator is obtained by using a contribution from $\Delta_{a b}^{h,(i)}$, and for the denominator, we include all the partonic channels.

In Fig. 3, we plot $R_{q g}^{(1)}$ for different choices of nonuniversal couplings, and we observe that the sign of the $q g$ subprocess crucially depends on the choice of couplings. Moreover, we find that $R_{q g}^{(1)}$ is positive and is as large as $70 \%$ for the couplings $(1.0,0.1)$, which explains the reason for the large $\mathrm{K}$-factor at the resonance region. However, the sign of the contribution from other subprocesses $q \bar{q}$ and $g g$ is found to be positive for various couplings.

In Fig. 4, we present the second order QCD corrections [at $\left(a_{s}^{2}\right)$ ] from various subprocesses to the dilepton production for the default choice of couplings $\left(k_{q}, k_{g}\right)=(0.5,1.0)$. Similar to the first order QCD corrections, the $g g$ subprocess has the dominant contribution over the rest while $q g$ has a negative contribution but is comparable in magnitude to that of $g g$. Because of this large $q g$ subprocess contribution which can flip its sign for certain couplings, it is necessary to study the percentage of its relative contribution $R_{q g}^{(2)}$ to the total second order correction. In Fig. 5, we present $R_{q g}^{(2)}$ for different choices of couplings. As can be seen from the figure, the $q g$

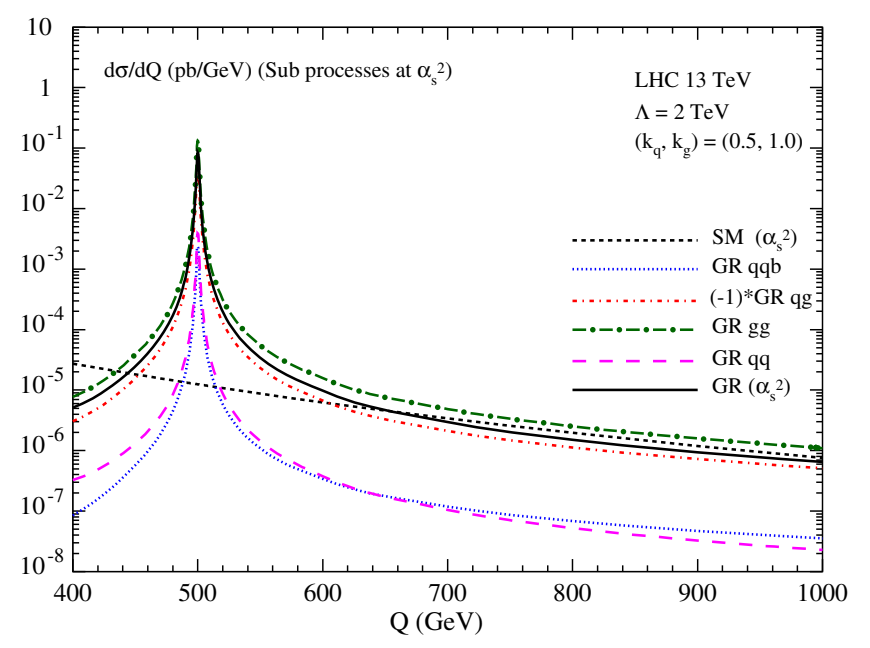

FIG. 4. Second order QCD corrections from various subprocesses to the dilepton invariant mass distribution.

contribution varies from about $-70 \%$ to about $35 \%$ for the choice of couplings considered here. In particular, for the couplings $(1.0,0.1)$ and $(0.5,0.1)$ the $q g$ contribution is positive while it is negative for the rest of the couplings as well as in the SM. This implies large K-factors for the choice of $(1.0,0.1)$ couplings for a wide range of the invariant mass distribution. It is worth mentioning here that in general the $q g$ subprocess has a negative contribution both in the SM and in the case of universal couplings, irrespective of the value of the latter.

We then present the dilepton invariant mass distribution to various orders in QCD for a particular choice of couplings $(1.0,0.5)$ in Fig. 6. In this case, the NLO QCD corrections for the signal (SM + spin-2) are as large as $60 \%$ while those at NNLO are about $80 \%$ at the resonance. Similar results are presented but for our default choice of model parameters in Fig. 7. Here, the

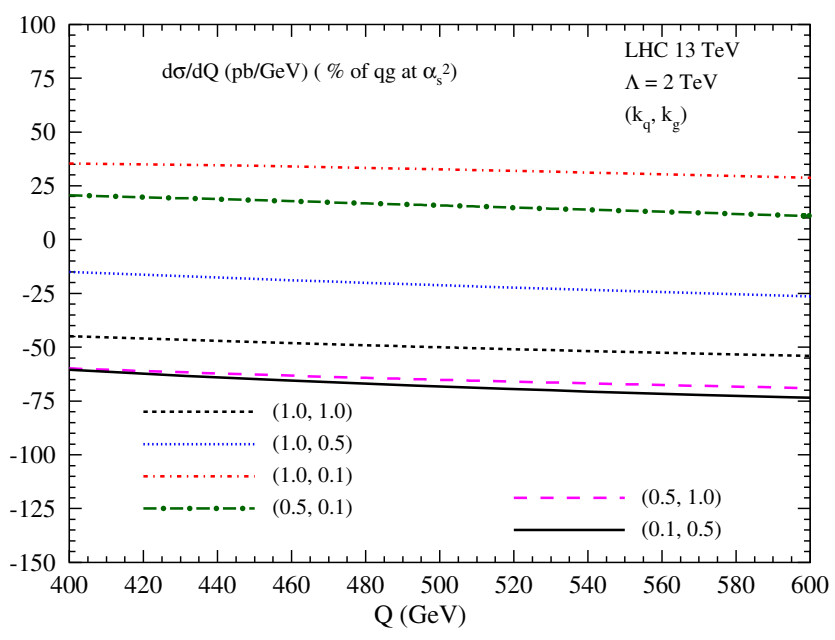

FIG. 5. Percentage of $q g$ contribution $R_{q g}^{(2)}$ as defined in the text. 

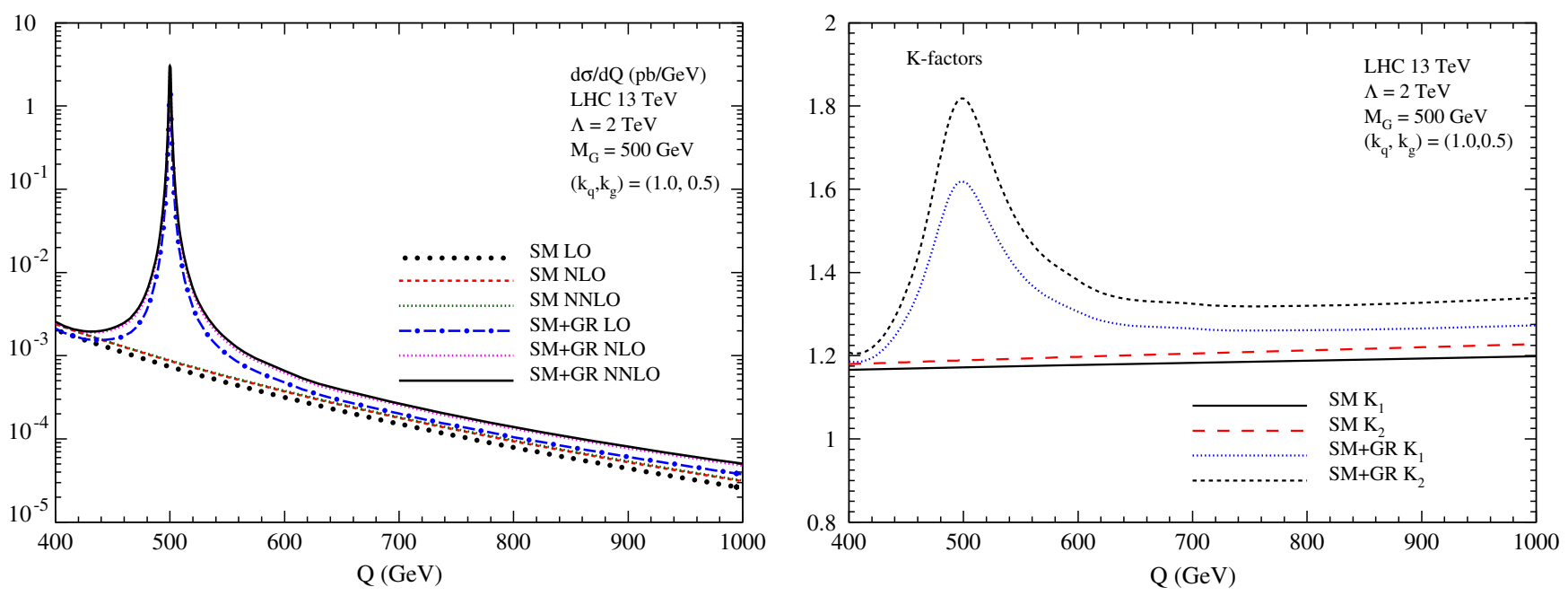

FIG. 6. Cross sections at different orders (left panel) and the corresponding K-factors $K_{1}$ and $K_{2}$ (right panel) are presented for different couplings.
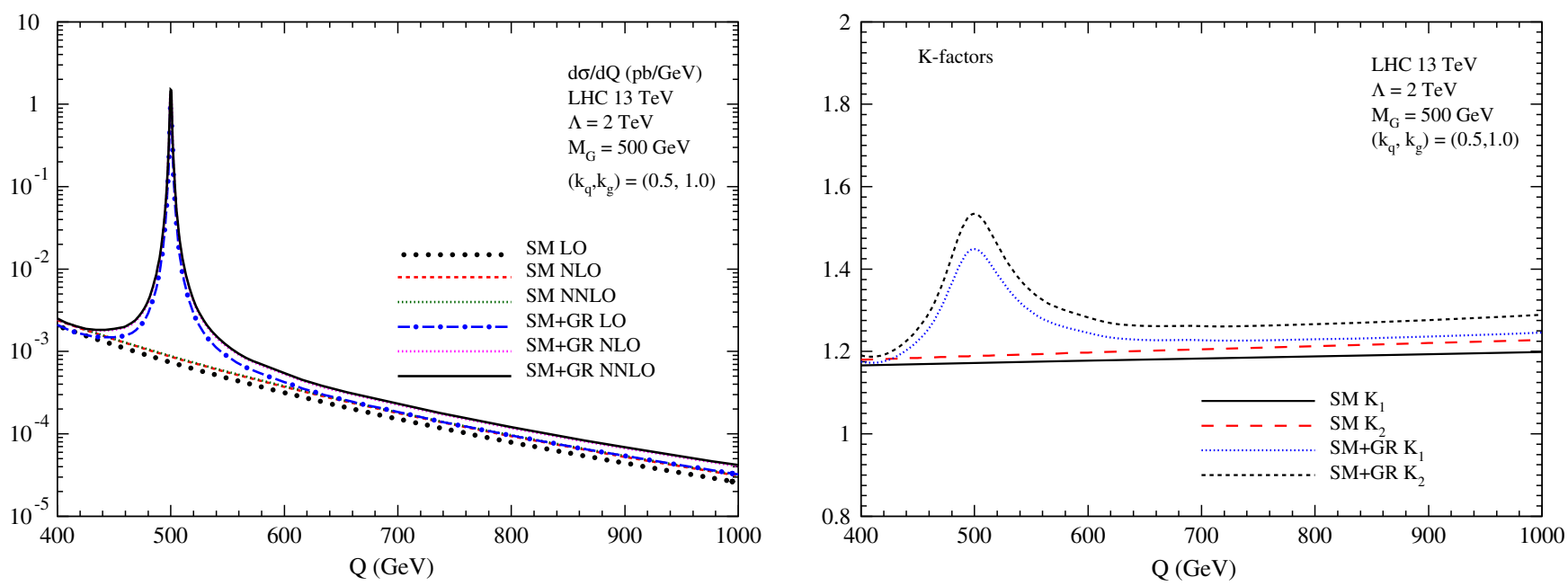

FIG. 7. Same as Fig. 6 but for a different set of couplings.

corresponding NLO corrections to the signal are about $45 \%$ while those of NNLO are about $55 \%$.

Next, we will study the invariant mass distributions of both the SM and the signal, in particular the impact of QCD corrections for different couplings. In Figs. 8, 9, and 10, we present these distributions in the left panel and the corresponding NNLO K-factors $\left(K_{2}\right)$ in the right panel for nine different sets of nonuniversal couplings. The respective $\mathrm{K}$-factors for the signal at the resonance region are found to vary from about 1.5 to about as large as 3.0, owing to different contributions from the $q g$ subprocess to the signal as explained before.

Further, we depict the dependence of invariant mass distributions to NNLO in QCD on the center of mass energy $\mathrm{E}_{\mathrm{cm}}$ of the protons at the LHC. We present our results for $\mathrm{E}_{\mathrm{cm}}=7,8,13$, and $14 \mathrm{TeV}$ energies for two different sets of couplings. In Fig. 11, we present the invariant mass distributions and the corresponding $\mathrm{K}$-factors for the universal couplings of $(1.0,1.0)$. For the default choice of nonuniversal couplings $(0.5,1.0)$, similar results are presented in Fig. 12. In both the cases, the $\mathrm{K}$-factors at the resonance region are found to be larger for the $7 \mathrm{TeV}$ case and are about 1.6.

In what follows, we study the renormalization scale $\mu_{R}$ and the factorization scale $\mu_{F}$ uncertainties in our predictions. For this, we define the ratios $R\left(\mu_{R}, \mu_{F}\right)$ of the invariant mass distributions computed at arbitrary scale to those computed at the fixed scale. These are defined as

$$
\mathrm{R}\left(\mu_{R}, \mu_{F}\right)=\frac{d \sigma\left(\mu_{R}, \mu_{F}\right) / d Q}{d \sigma\left(Q_{0}, Q_{0}\right) / d Q}
$$

For a systematic study of these scale uncertainties, we use LO (NLO and NNLO) PDFs for LO (NLO and NNLO) 

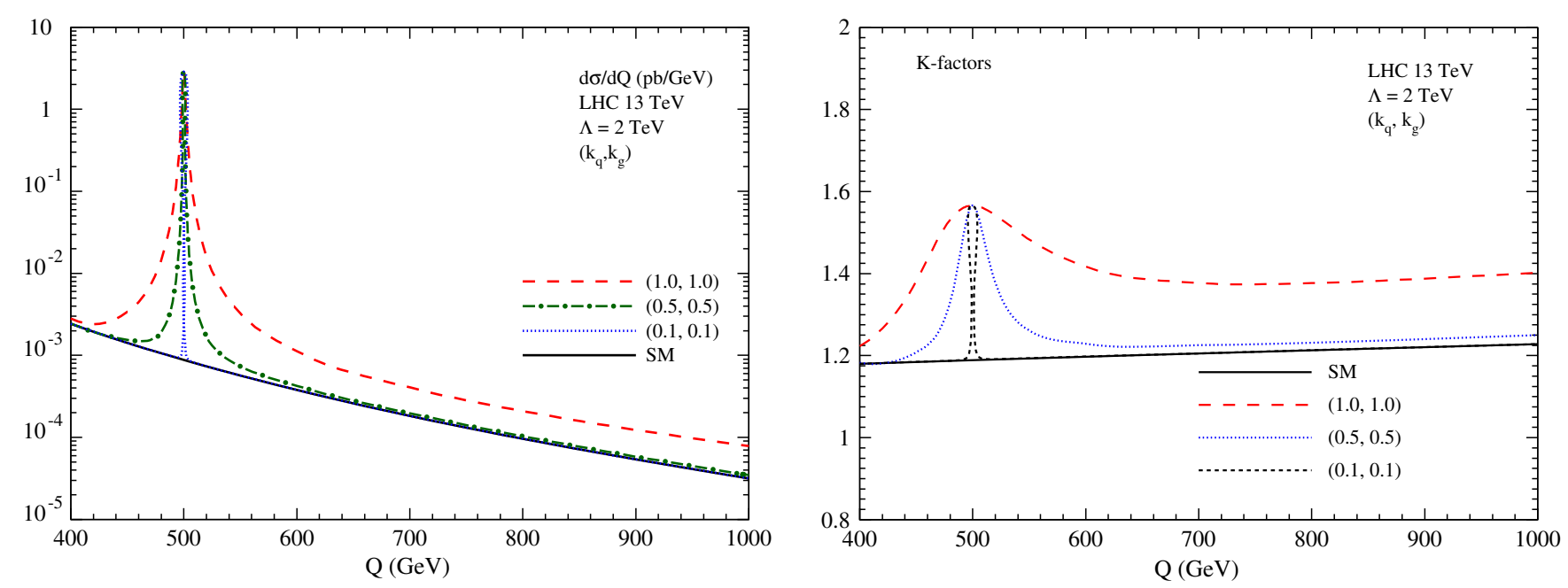

FIG. 8. Dilepton invariant mass distributions to NNLO for different choices of couplings (left panel) and the corresponding K-factors (right panel) are presented.
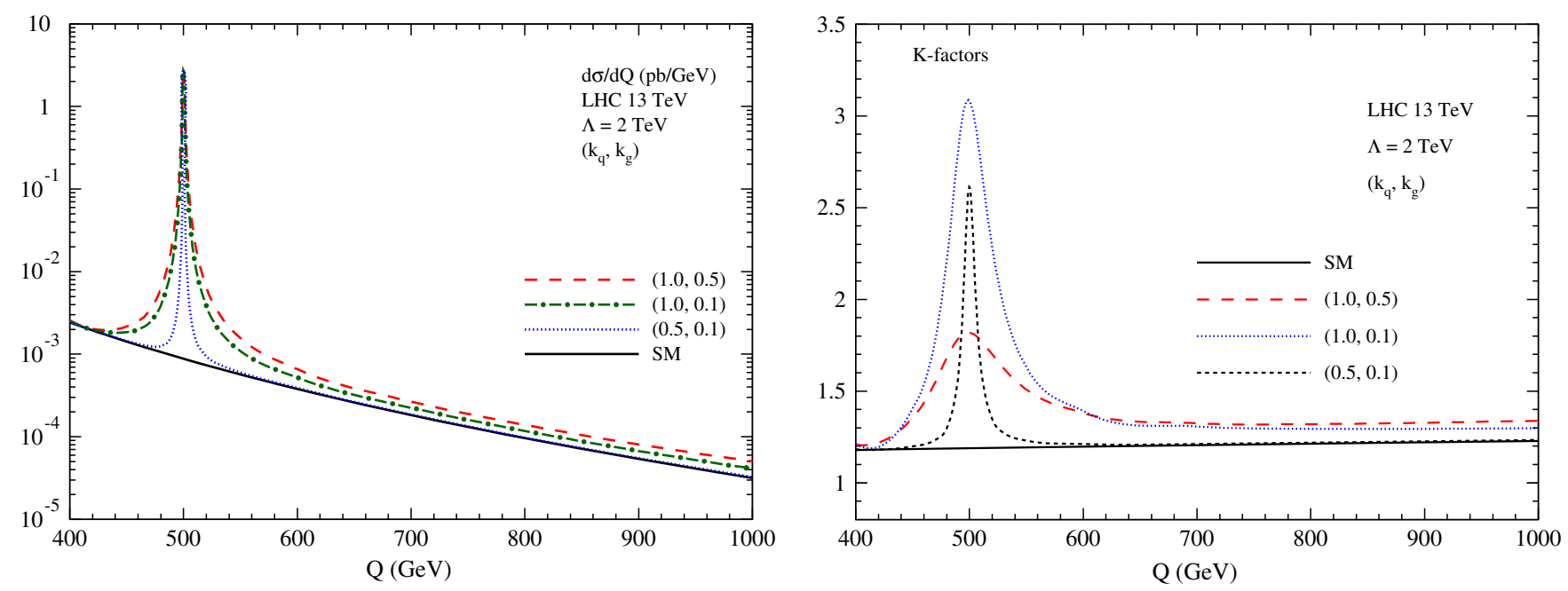

FIG. 9. Same as Fig. 8 but for a different set of couplings.
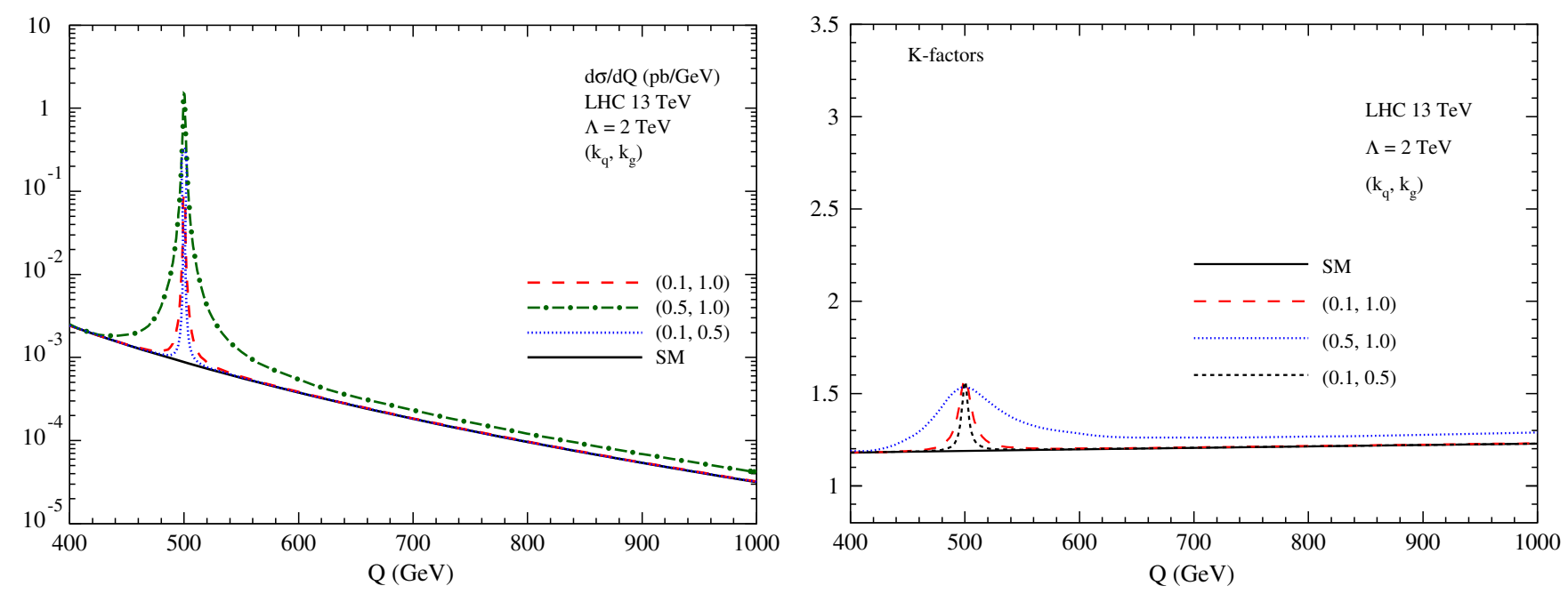

FIG. 10. Same as Fig. 8 but for a different set of couplings. 

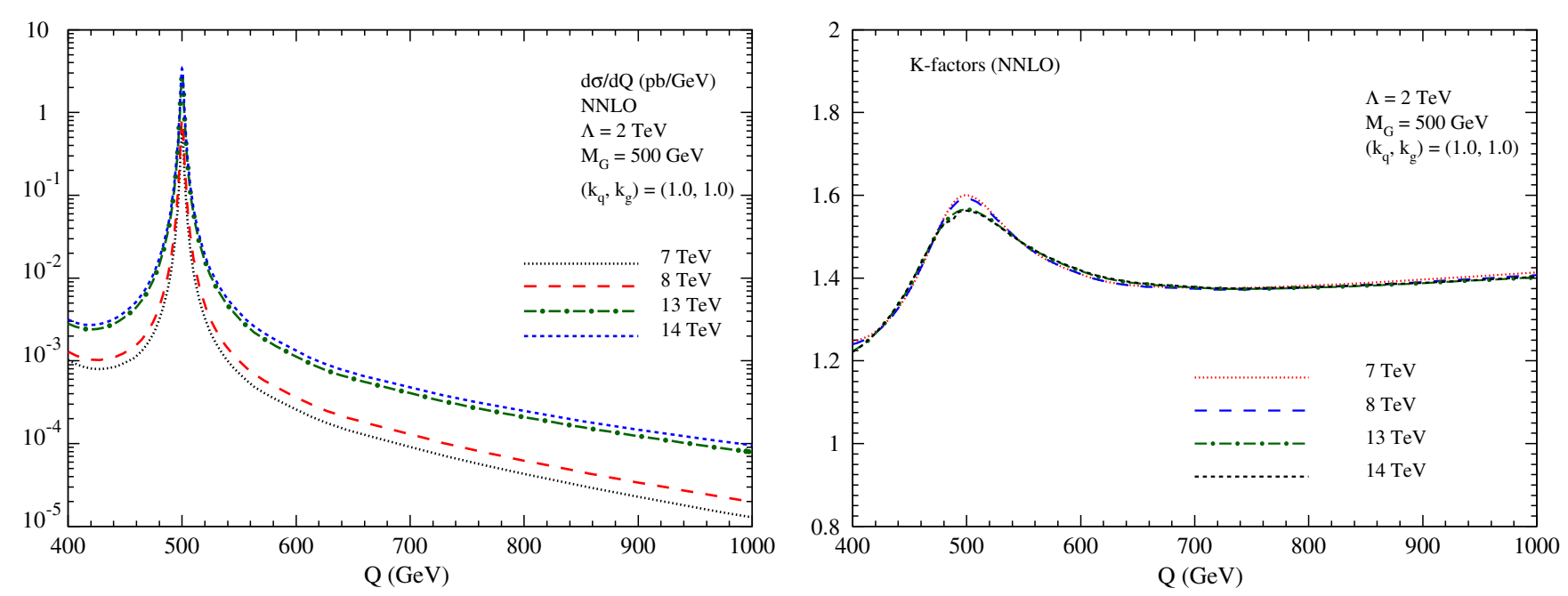

FIG. 11. Dependence of cross sections on the dilepton invariant mass distribution for universal couplings (1.0,1.0).
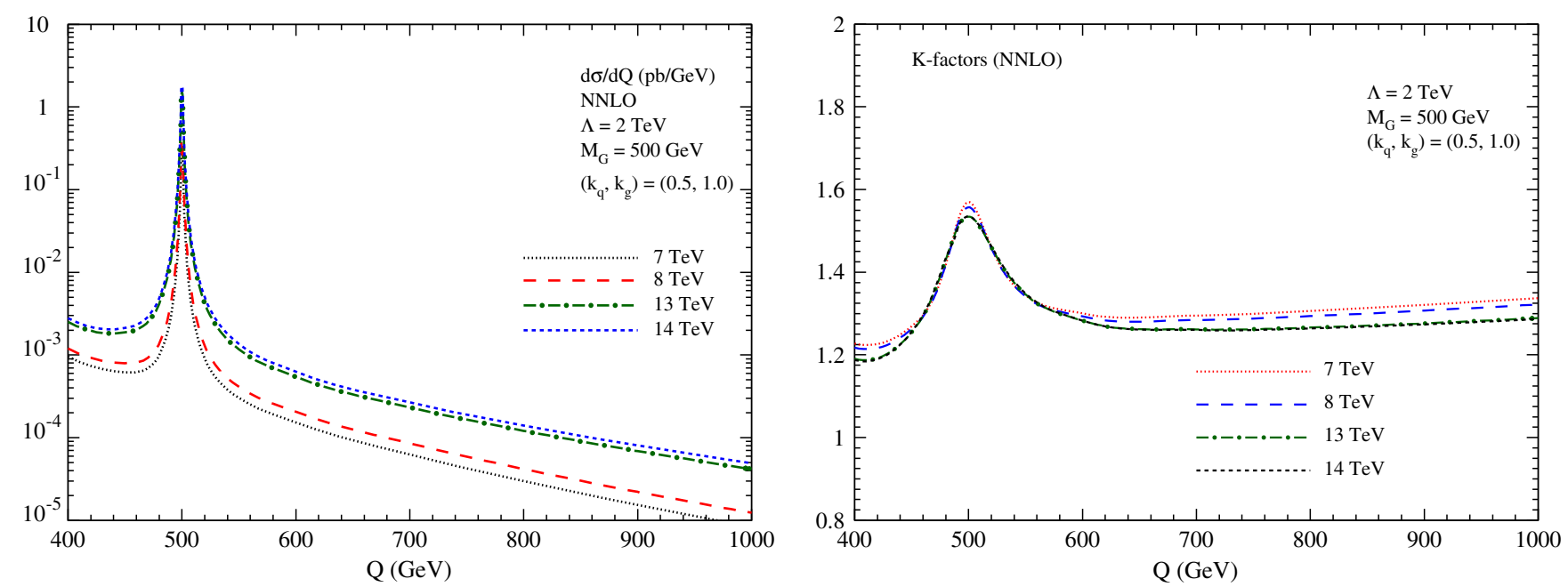

FIG. 12. Same as Fig. 11 but for the default choice of nonuniversal couplings $(0.5,1.0)$.
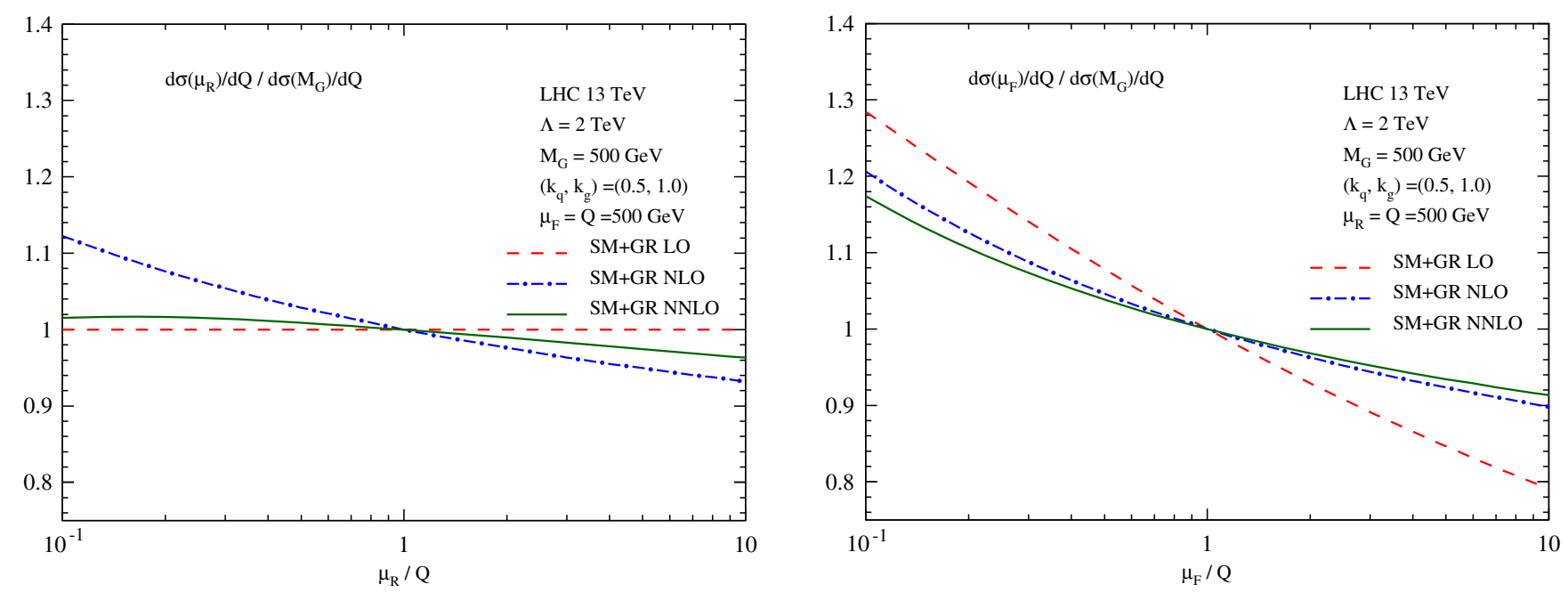

FIG. 13. Renormalization (left) and factorization (right) scale dependence of the dilepton invariant mass distribution at LO, NLO, and NNLO. 


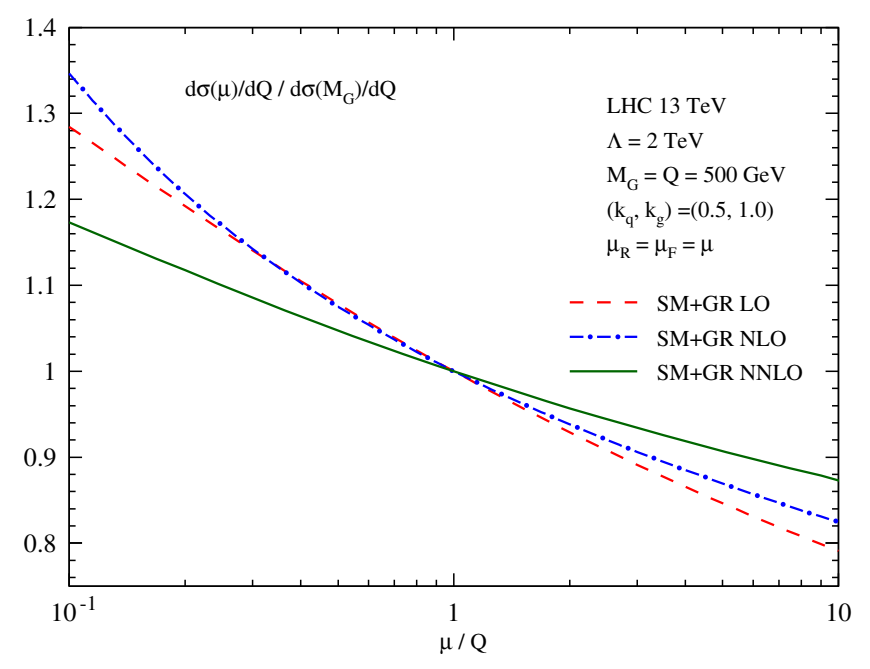

FIG. 14. Same as Fig. 13 but with $\mu_{R}=\mu_{F}=\mu$.

cross sections respectively. For convenience, we will study at the resonance region, i.e., $Q=M=500 \mathrm{GeV}$. The fixed scale is set equal to $Q_{0}=M$. In the left panel of Fig. 13, we present $\mathrm{R}\left(\mu_{R}, Q_{0}\right)$ by varying $\mu_{R}$ from $0.1 Q$ to $10 Q$ and keeping $\mu_{F}=Q_{0}$ fixed. At LO, there is no scale $\mu_{R}$ entering the cross section. The corresponding scale uncertainties at NLO and NNLO are, respectively, about $19 \%$ and $5 \%$.

In the right panel of Fig. 13, we present $\mathrm{R}\left(Q_{0}, \mu_{F}\right)$ by varying $\mu_{F}$ from $0.1 Q$ to $10 Q$ and keeping $\mu_{R}=Q_{0}$ fixed. For this range of factorization scale variation, the uncertainties in the distributions at LO, NLO, and NNLO are, respectively, about $49 \%, 31 \%$, and $26 \%$.

Finally, we present $\mathrm{R}(\mu, \mu)$ where $\left(\mu_{R}=\mu_{F}=\mu\right)$ in Fig. 14 by varying $\mu$ from $0.1 Q$ to $10 Q$. The corresponding scale uncertainties at LO, NLO, and NNLO are, respectively, about $49 \%, 52 \%$, and $30 \%$.

Before we summarize, we also study the uncertainties in our predictions due to different choices of PDFs used in the

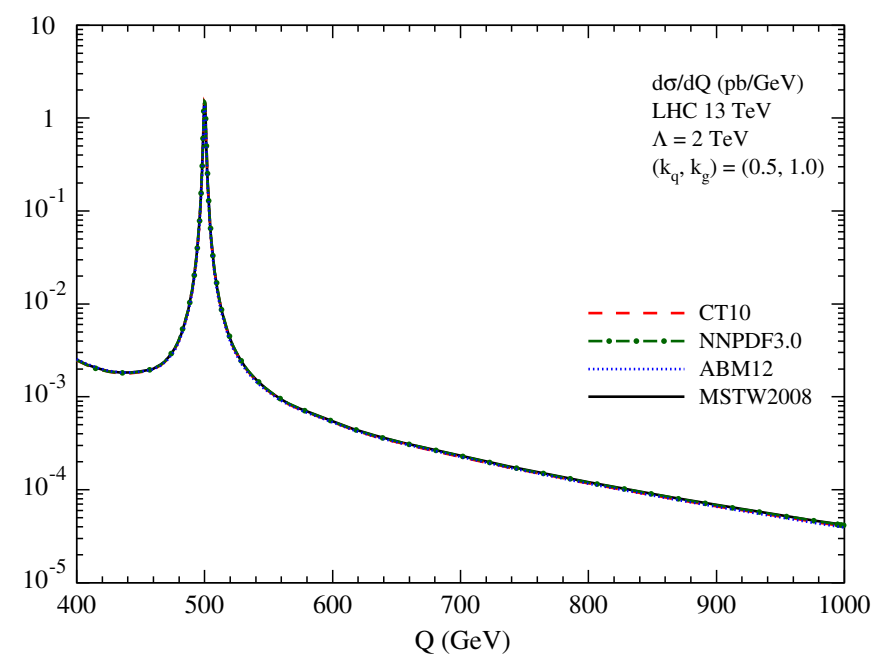

calculation. For this analysis, we make predictions using MSTW2008, CT10, NNPDF3.0, and ABM12 PDFs. The results for the invariant mass distributions for the signal at NNLO are presented in the left panel of Fig. 15 and the corresponding K-factors are presented in the right panel of Fig. 15. The K-factors here are found to vary from 1.18 at $Q=400 \mathrm{GeV}$ to about 1.28 at $Q=1000 \mathrm{GeV}$, while at the resonance they are about 1.54 .

\section{CONCLUSION}

In this article, we have studied for the first time to our knowledge the impact of NNLO QCD corrections to the production of a pair of leptons in the presence of a massive spin-2 particle at the LHC. This is done in a minimal scenario where spin-2 particles couple differently to SM fermions and SM bosons. This task has been achieved by using the universal IR structure of QCD amplitudes and the additional UV renormalization that is particularly required for the case of nonuniversal couplings, thanks to the recent computations of the form factors in QCD beyond leading order with nonuniversal couplings.

Unlike the models with universal couplings, here the phenomenology is rich and different. For collider phenomenology at the LHC, we present the results for the dilepton production via spin-2 particle in particular for the invariant mass distribution of a pair of leptons for LHC energies. Even at LO, one can notice that the signal has different cross sections at the resonance region in contrast to the gravity mediated models where the signal has the same cross section for different universal couplings. At higher orders in QCD, say NLO onwards, the spin-2 exploits its freedom of being produced with different coupling strengths even for a given subprocess. This particular aspect here makes the QCD radiative corrections crucially dependent on the choice of the spin-2 coupling strength. Hence the impact of QCD corrections here is very much

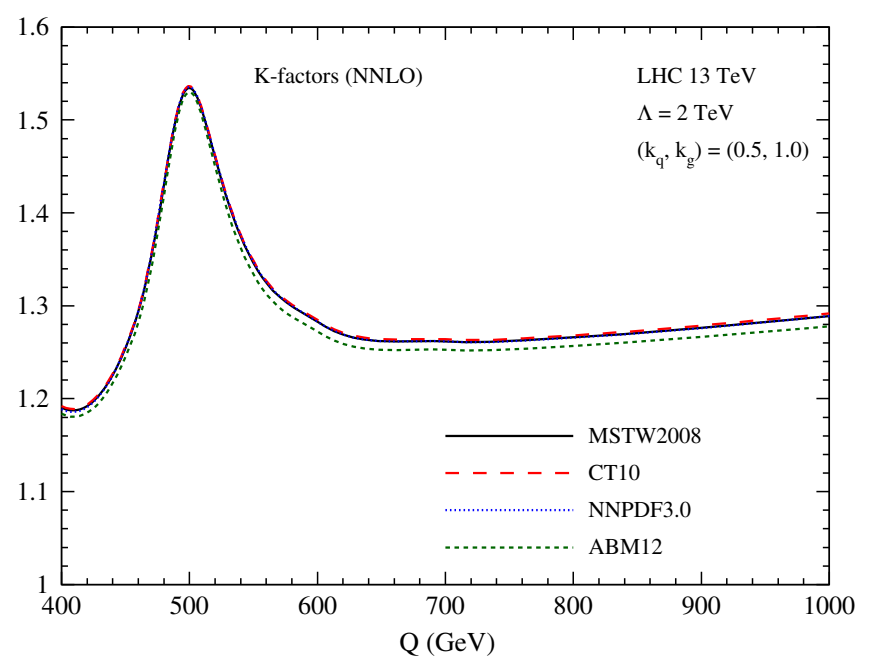

FIG. 15. Dilepton invariant mass distributions for different choices of PDFs. 
different from those of dilepton or Higgs production in the SM.

We find from our numerical results that the QCD corrections for $\left(k_{q}, k_{g}\right)=(1.0,0.1)$ are dominant over the rest of the choice of couplings, making the K-factors as large as 2.5 or more. For this choice of couplings, the LO gluon fusion contribution is very small although gluon fluxes are high for the kinematic region of producing a $500 \mathrm{GeV}$ particle. But at higher orders where the spin-2 can be emitted from a quark line with large coupling strength, the large quark-gluon fluxes at LHC energies can potentially enhance the spin- 2 production rate, as is evident from the numerical results. For dilepton production the "sign" of the $q g$ subprocess is usually negative both in the SM and in the models of universal couplings. But here we note that the sign of the $q g$ subprocess contribution changes with the nonuniversal couplings and for the above choice it is positive.

We also gave predictions for different center of mass energies of the incoming protons at the LHC and found that the K-factors are larger for the $7 \mathrm{TeV}$ case. We further quantified the renormalization and factorization scale uncertainties. For the variation of the scales $\mu_{R}$ and $\mu_{F}$ between $0.1 Q$ and $10 Q$, the uncertainties are found to get reduced from about $50 \%$ at $\mathrm{LO}$ to about $30 \%$ at NNLO. For completeness, we also quantified the uncertainty in our predictions due to different choice of the PDFs.

These NNLO QCD predictions for the hadroproduction of a massive spin-2 with nonuniversal couplings will augment the similar results previously computed at the NLO level and complement the earlier results for NNLO QCD corrections in models with spin-2/graviton universal couplings.

\section{ACKNOWLEDGMENTS}

We thank G. Das for helping us to validate a part of the numerical results. We also thank T. Ahmed and N. Rana for useful discussions. V. R. thanks M. Neubert for the visit at University of Mainz where the last part of the work was carried out.

\section{APPENDIX: RENORMALIZED FORM FACTORS}

We present here the results for the renormalized form factors [11] that are used in the present computation. In the color space, the UV renormalized matrix elements of composite operators $\mathcal{O}^{I}, I=G, Q$ between a pair of onshell partonic states $i=q, g$ and the vacuum state are expanded in powers of coupling constant $a_{s}$ as

$$
\left|\mathcal{M}_{i}^{I}\right\rangle=\sum_{n=0}^{\infty} a_{s}^{n}\left|\mathcal{M}_{i}^{I,(n)}\right\rangle,
$$

where $i=q, \bar{q}, g$. The on-shell form factor of $\hat{\mathcal{O}}^{I}, I=G, Q$ is defined by taking the overlap of $\left|\mathcal{M}_{i}^{I}\right\rangle$ with its leading order amplitude normalized with respect to the leading order contribution. We find that there are four independent form factors:

$$
\begin{aligned}
\mathcal{F}^{I, g,(n)} & =\frac{\left\langle\mathcal{M}_{g}^{G,(0)} \mid \mathcal{M}_{g}^{I,(n)}\right\rangle}{\left\langle\mathcal{M}_{g}^{G,(0)} \mid \mathcal{M}_{g}^{G,(0)}\right\rangle}, \\
\mathcal{F}^{I, q,(n)} & =\frac{\left\langle\mathcal{M}_{q}^{Q,(0)} \mid \mathcal{M}_{q}^{I,(n)}\right\rangle}{\left\langle\mathcal{M}_{q}^{Q,(0)} \mid \mathcal{M}_{q}^{Q,(0)}\right\rangle}, \quad I=G, Q .
\end{aligned}
$$

Note that the nondiagonal amplitudes, i.e., $\left|\mathcal{M}_{g}^{Q,(n)}\right\rangle$ and $\left|\mathcal{M}_{q}^{G,(n)}\right\rangle$, start at the one-loop level, and hence, the corresponding form factors start at $\mathcal{O}\left(a_{s}\right)$. The relevant UV renormalized form factors at the two-loop level are given below:

$$
\begin{aligned}
& \mathcal{F}^{G, g,(1)}= \frac{1}{\epsilon^{2}} C_{A}[-8]+\frac{1}{\epsilon}\left[C_{A}\left(\frac{22}{3}\right)+n_{f}\left(-\frac{4}{3}\right)\right]+C_{A}\left[-\frac{203}{18}+\zeta_{2}\right]+\epsilon C_{A}\left[\frac{2879}{216}-\frac{7}{3} \zeta_{3}-\frac{11}{12} \zeta_{2}\right] \\
&+\epsilon^{2} C_{A}\left[-\frac{37307}{2592}+\frac{77}{36} \zeta_{3}+\frac{203}{144} \zeta_{2}+\frac{47}{80} \zeta_{2}^{2}\right], \\
& \mathcal{F}^{G, g,(2)}= \frac{1}{\epsilon^{4}} C_{A}^{2}[32]+\frac{1}{\epsilon^{3}}\left[C_{A}^{2}\left(-\frac{308}{3}\right)+n_{f} C_{A}\left(\frac{56}{3}\right)\right]+\frac{1}{\epsilon^{2}}\left[C_{A}^{2}\left(\frac{1162}{9}-4 \zeta_{2}\right)+n_{f} C_{A}\left(-\frac{52}{3}\right)+n_{f}^{2}\left(\frac{16}{9}\right)\right] \\
&+ \frac{1}{\epsilon}\left[C_{A}^{2}\left(-\frac{4420}{27}+\frac{50}{3} \zeta_{3}+11 \zeta_{2}\right)+n_{f} C_{A}\left(\frac{278}{27}-\frac{2}{3} \zeta_{2}\right)+n_{f} C_{F}(-2)\right] \\
&+ C_{A}^{2}\left(\frac{11854}{81}-\frac{253}{9} \zeta_{3}-\frac{94}{9} \zeta_{2}-\frac{21}{5} \zeta_{2}^{2}\right)+n_{f} C_{A}\left(\frac{1105}{162}+2 \zeta_{3}-\frac{26}{9} \zeta_{2}\right)+n_{f} C_{F}\left(\frac{1049}{162}-\frac{16}{9} \zeta_{2}\right), \\
& \mathcal{F}^{G, q,(1)}=C_{F}\left(\frac{34}{9}\right)+\epsilon C_{F}\left(-\frac{79}{27}+\frac{2}{3} \zeta_{2}\right)+\epsilon^{2} C_{F}\left(\frac{401}{162}-\frac{14}{9} \zeta_{3}-\frac{17}{36} \zeta_{2}\right),
\end{aligned}
$$




$$
\begin{aligned}
& \mathcal{F}^{G, q,(2)}=\frac{1}{\epsilon^{2}} C_{F}^{2}\left(-\frac{272}{9}\right)+\frac{1}{\epsilon} C_{F}^{2}\left(\frac{1244}{27}-\frac{16}{3} \zeta_{2}\right)+C_{F} C_{A}\left(\frac{3913}{81}+\frac{28}{9} \zeta_{2}\right)+C_{F}^{2}\left(-\frac{2603}{27}+\frac{112}{9} \zeta_{3}+\frac{56}{3} \zeta_{2}\right) \\
& +n_{f} C_{F}\left(-\frac{1166}{81}\right) \\
& \mathcal{F}^{Q, g,(1)}=n_{f}\left(\frac{35}{18}\right)+\epsilon n_{f}\left(-\frac{497}{216}+\frac{1}{6} \zeta_{2}\right)+\epsilon^{2} n_{f}\left(\frac{6593}{2592}-\frac{7}{18} \zeta_{3}-\frac{35}{144} \zeta_{2}\right) \\
& \mathcal{F}^{Q, g,(2)}=\frac{1}{\epsilon^{2}} n_{f} C_{A}\left(-\frac{140}{9}\right)+\frac{1}{\epsilon}\left[n_{f} C_{A}\left(\frac{98}{3}-\frac{4}{3} \zeta_{2}\right)+n_{f}^{2}\left(-\frac{70}{27}\right)\right]+n_{f} C_{A}\left(-\frac{7625}{162}+\frac{100}{9} \zeta_{3}+\frac{53}{9} \zeta_{2}\right) \\
& +n_{f} C_{F}\left(\frac{299}{81}-8 \zeta_{3}+\frac{16}{9} \zeta_{2}\right)+n_{f}^{2}\left(\frac{497}{162}-\frac{2}{9} \zeta_{2}\right) \\
& \mathcal{F}^{Q, q,(1)}=\frac{1}{\epsilon^{2}} C_{F}(-8)+\frac{1}{\epsilon} C_{F}(6)+C_{F}\left(-\frac{124}{9}+\zeta_{2}\right)+\epsilon C_{F}\left(\frac{403}{27}-\frac{7}{3} \zeta_{3}-\frac{17}{12} \zeta_{2}\right) \\
& +\epsilon^{2} C_{F}\left(-\frac{2507}{162}+\frac{119}{36} \zeta_{3}+\frac{31}{18} \zeta_{2}+\frac{47}{80} \zeta_{2}^{2}\right), \\
& \mathcal{F}^{Q, q,(2)}=\frac{1}{\epsilon^{4}} C_{F}^{2}(32)+\frac{1}{\epsilon^{3}}\left[C_{F} C_{A}(-44)+C_{F}^{2}(-48)+n_{f} C_{F}(8)\right] \\
& +\frac{1}{\epsilon^{2}}\left[C_{F} C_{A}\left(\frac{64}{9}+4 \zeta_{2}\right)+C_{F}^{2}\left(\frac{1154}{9}-8 \zeta_{2}\right)+n_{f} C_{F}\left(-\frac{16}{9}\right)\right] \\
& +\frac{1}{\epsilon}\left[C_{F} C_{A}\left(\frac{961}{54}-26 \zeta_{3}+11 \zeta_{2}\right)+C_{F}^{2}\left(-\frac{10831}{54}+\frac{128}{3} \zeta_{3}+\frac{16}{3} \zeta_{2}\right)+n_{f} C_{F}\left(-\frac{65}{27}-2 \zeta_{2}\right)\right] \\
& +C_{F} C_{A}\left(-\frac{31495}{216}+\frac{601}{9} \zeta_{3}-\frac{73}{2} \zeta_{2}+\frac{44}{5} \zeta_{2}^{2}\right)+C_{F}^{2}\left(\frac{68677}{216}-\frac{922}{9} \zeta_{3}+\frac{11}{6} \zeta_{2}-13 \zeta_{2}^{2}\right) \\
& \left.+n_{f} C_{F}\left(\frac{9469}{324}+\frac{2}{9} \zeta_{3}+\frac{47}{9} \zeta_{2}\right)\right] \text {. }
\end{aligned}
$$

[1] G. Aad et al. (ATLAS Collaboration), Observation of a new particle in the search for the Standard Model Higgs boson with the ATLAS detector at the LHC, Phys. Lett. B 716, 1 (2012).

[2] S. Chatrchyan et al. (CMS Collaboration), Observation of a new boson at a mass of $125 \mathrm{GeV}$ with the CMS experiment at the LHC, Phys. Lett. B 716, 30 (2012).

[3] R. V. Harlander and W. B. Kilgore, Next-to-Next-to-Leading Order Higgs Production at Hadron Colliders, Phys. Rev. Lett. 88, 201801 (2002).

[4] C. Anastasiou and K. Melnikov, Higgs boson production at hadron colliders in NNLO QCD, Nucl. Phys. B646, 220 (2002).

[5] V. Ravindran, J. Smith, and W. L. van Neerven, NNLO corrections to the total cross-section for Higgs boson production in hadron hadron collisions, Nucl. Phys. B665, 325 (2003).
[6] G. Degrassi, S. Di Vita, J. Elias-Miró, J. R. Espinosa, G. F. Giudice, G. Isidori, and A. Strumia, Higgs mass and vacuum stability in the Standard Model at NNLO, J. High Energy Phys. 08 (2012) 098.

[7] L. Randall and R. Sundrum, A Large Mass Hierarchy from a Small Extra Dimension, Phys. Rev. Lett. 83, 3370 (1999).

[8] V. Khachatryan et al. (CMS Collaboration), Search for highmass diphoton resonances in proton-proton collisions at $13 \mathrm{TeV}$ and combination with $8 \mathrm{TeV}$ search, Phys. Lett. B 767, 147 (2017).

[9] M. Aaboud et al. (ATLAS Collaboration), Search for diboson resonances with boson-tagged jets in $p p$ collisions at $\sqrt{s}=13 \mathrm{TeV}$ with the ATLAS detector, Phys. Lett. B 777, 91 (2018).

[10] P. Artoisenet et al., A framework for Higgs characterisation, J. High Energy Phys. 11 (2013) 043. 
[11] T. Ahmed, P. Banerjee, P. K. Dhani, P. Mathews, N. Rana, and V. Ravindran, Three loop form factors of a massive spin-2 particle with nonuniversal coupling, Phys. Rev. D 95, 034035 (2017).

[12] N. K. Nielsen, The energy momentum tensor in a nonabelian quark gluon theory, Nucl. Phys. B120, 212 (1977).

[13] P. Mathews, V. Ravindran, K. Sridhar, and W. L. van Neerven, Next-to-leading order QCD corrections to the Drell-Yan cross section in models of TeV-scale gravity, Nucl. Phys. B713, 333 (2005).

[14] P. Mathews and V. Ravindran, Angular distribution of DrellYan process at hadron colliders to NLO-QCD in models of TeV scale gravity, Nucl. Phys. B753, 1 (2006).

[15] M. C. Kumar, P. Mathews, and V. Ravindran, PDF and scale uncertainties of various DY distributions in ADD and RS models at hadron colliders, Eur. Phys. J. C 49, 599 (2007).

[16] M. C. Kumar, P. Mathews, V. Ravindran, and A. Tripathi, Diphoton signals in theories with large extra dimensions to NLO QCD at hadron colliders, Phys. Lett. B 672, 45 (2009).

[17] M. C. Kumar, P. Mathews, V. Ravindran, and A. Tripathi, Direct photon pair production at the LHC to order $\alpha_{s}$ in TeV scale gravity models, Nucl. Phys. B818, 28 (2009).

[18] N. Agarwal, V. Ravindran, V. K. Tiwari, and A. Tripathi, Z boson pair production at the LHC to $O\left(\alpha_{s}\right)$ in $\mathrm{TeV}$ scale gravity models, Nucl. Phys. B830, 248 (2010).

[19] N. Agarwal, V. Ravindran, V. K. Tiwari, and A. Tripathi, Next-to-leading order QCD corrections to the $Z$ boson pair production at the LHC in Randall Sundrum model, Phys. Lett. B 686, 244 (2010).

[20] N. Agarwal, V. Ravindran, V. K. Tiwari, and A. Tripathi, $W^{+} W^{-}$production in large extra dimension model at nextto-leading order in QCD at the LHC, Phys. Rev. D 82, 036001 (2010).

[21] N. Agarwal, V. Ravindran, V. K. Tiwari, and A. Tripathi, Next-to-leading order QCD corrections to $W^{+} W^{-}$production at the LHC in Randall Sundrum model, Phys. Lett. B 690, 390 (2010).

[22] R. Frederix, M. K. Mandal, P. Mathews, V. Ravindran, S. Seth, P. Torrielli, and M. Zaro, Diphoton production in the ADD model to NLO + parton shower accuracy at the LHC, J. High Energy Phys. 12 (2012) 102.

[23] R. Frederix, M. K. Mandal, P. Mathews, V. Ravindran, and S. Seth, Drell-Yan, $Z Z, W^{+} W^{-}$production in SM \& ADD model to NLO + PS accuracy at the LHC, Eur. Phys. J. C 74, 2745 (2014).

[24] G. Das, P. Mathews, V. Ravindran, and S. Seth, RS resonance in di-final state production at the LHC to NLO + PS accuracy, J. High Energy Phys. 10 (2014) 188.

[25] G. Das, C. Degrande, V. Hirschi, F. Maltoni, and H.-S. Shao, NLO predictions for the production of a $(750 \mathrm{GeV})$ spin-two particle at the LHC, Phys. Lett. B 770, 507 (2017).

[26] D. de Florian, M. Mahakhud, P. Mathews, J. Mazzitelli, and V. Ravindran, Quark and gluon spin-2 form factors to twoloops in QCD, J. High Energy Phys. 02 (2014) 035.

[27] D. de Florian, M. Mahakhud, P. Mathews, J. Mazzitelli, and V. Ravindran, Next-to-next-to-leading order QCD corrections in models of TeV-scale gravity, J. High Energy Phys. 04 (2014) 028.
[28] T. Ahmed, P. Banerjee, P. K. Dhani, M. C. Kumar, P. Mathews, N. Rana, and V. Ravindran, NNLO QCD corrections to the Drell-Yan cross section in models of TeVscale gravity, Eur. Phys. J. C 77, 22 (2017).

[29] T. Ahmed, M. Mahakhud, P. Mathews, N. Rana, and V. Ravindran, Two-loop QCD correction to massive spin-2 resonance $\rightarrow 3$ gluons, J. High Energy Phys. 05 (2014) 107.

[30] T. Ahmed, G. Das, P. Mathews, N. Rana, and V. Ravindran, The two-loop QCD correction to massive spin-2 resonance $\rightarrow q \bar{q} g$, Eur. Phys. J. C 76, 667 (2016).

[31] G. Aad et al. (ATLAS Collaboration), Study of the spin and parity of the Higgs boson in diboson decays with the ATLAS detector, Eur. Phys. J. C 75, 476 (2015).

[32] L.E. Pedersen, Probing the nature of the Higgs boson: A study of the Higgs spin and parity through the $Z Z^{*} \rightarrow 4 l$ final state at the ATLAS Experiment, Ph.D. thesis, Bohr Institute, 2015.

[33] T. Han, J. D. Lykken, and R.-J. Zhang, On Kaluza-Klein states from large extra dimensions, Phys. Rev. D 59, 105006 (1999).

[34] P. Mathews, V. Ravindran, and K. Sridhar, NLO*_QCD corrections to $e+e-\rightarrow$ hadrons in models of $\mathrm{TeV}$-scale gravity, J. High Energy Phys. 08 (2004) 048.

[35] G. Altarelli, R. K. Ellis, and G. Martinelli, Leptoproduction and Drell-Yan processes beyond the leading approximation in chromodynamics, Nucl. Phys. B143, 521 (1978).

[36] T. Matsuura and W. L. van Neerven, Second order logarithmic corrections to the Drell-Yan cross-section, Z. Phys. C 38, 623 (1988).

[37] T. Matsuura, S. C. van der Marck, and W. L. van Neerven, The calculation of the second order soft and virtual contributions to the Drell-Yan cross-section, Nucl. Phys. B319, 570 (1989).

[38] R. Hamberg, W. L. van Neerven, and T. Matsuura, A complete calculation of the order $\alpha-s^{2}$ correction to the Drell-Yan $K$ factor, Nucl. Phys. B359, 343 (1991).

[39] T. Ahmed, M. Mahakhud, N. Rana, and V. Ravindran, DrellYan Production at Threshold to Third Order in QCD, Phys. Rev. Lett. 113, 112002 (2014).

[40] F. V. Tkachov, A theorem on analytical calculability of four loop renormalization group functions, Phys. Lett. 100B, 65 (1981).

[41] K. Chetyrkin and F. Tkachov, Integration by parts: The algorithm to calculate beta functions in 4 loops, Nucl. Phys. B192, 159 (1981).

[42] T. Gehrmann and E. Remiddi, Differential equations for two loop four point functions, Nucl. Phys. B580, 485 (2000).

[43] C. Anastasiou, C. Duhr, F. Dulat, E. Furlan, T. Gehrmann, F. Herzog, and B. Mistlberger, Higgs boson gluon-fusion production at threshold in $\mathrm{N}^{3} \mathrm{LO} \mathrm{QCD}$, Phys. Lett. B 737, 325 (2014).

[44] C. Anastasiou, C. Duhr, F. Dulat, F. Herzog, and B. Mistlberger, Higgs Boson Gluon-Fusion Production in QCD at Three Loops, Phys. Rev. Lett. 114, 212001 (2015).

[45] C. Anastasiou, C. Duhr, F. Dulat, E. Furlan, T. Gehrmann, F. Herzog, A. Lazopoulos, and B. Mistlberger, High precision determination of the gluon fusion Higgs boson cross-section at the LHC, J. High Energy Phys. 05 (2016) 058.

[46] T. Kinoshita, Mass singularities of Feynman amplitudes, J. Math. Phys. (N.Y.) 3, 650 (1962). 
[47] T. D. Lee and M. Nauenberg, Degenerate systems and mass singularities, Phys. Rev. 133, B1549 (1964).

[48] D. J. Gross and F. Wilczek, Ultraviolet Behavior of Nonabelian Gauge Theories, Phys. Rev. Lett. 30, 1343 (1973).

[49] H. D. Politzer, Reliable Perturbative Results for Strong Interactions?, Phys. Rev. Lett. 30, 1346 (1973).

[50] W. E. Caswell, Asymptotic Behavior of Nonabelian Gauge Theories to Two Loop Order, Phys. Rev. Lett. 33, 244 (1974).

[51] O. V. Tarasov and A. A. Vladimirov, Three loop calculations in non-Abelian gauge theories, Phys. Part. Nucl. 44, 791 (2013).

[52] S. A. Larin and J. A. M. Vermaseren, The three loop QCD beta function and anomalous dimensions, Phys. Lett. B 303, 334 (1993).

[53] G. Altarelli and G. Parisi, Asymptotic freedom in parton language, Nucl. Phys. B126, 298 (1977).
[54] E. G. Floratos, R. Lacaze, and C. Kounnas, Space and timelike cut vertices in QCD beyond the leading order. 2. The singlet sector, Phys. Lett. 98B, 285 (1981).

[55] E. G. Floratos, R. Lacaze, and C. Kounnas, Space and timelike cut vertices in QCD beyond the leading order. 1. Nonsinglet sector, Phys. Lett. 98B, 89 (1981).

[56] G. Curci, W. Furmanski, and R. Petronzio, Evolution of parton densities beyond leading order: The nonsinglet case, Nucl. Phys. B175, 27 (1980).

[57] S. Moch, J. A. M. Vermaseren, and A. Vogt, The three loop splitting functions in QCD: The nonsinglet case, Nucl. Phys. B688, 101 (2004).

[58] A. Vogt, S. Moch, and J. A. M. Vermaseren, The three-loop splitting functions in QCD: The singlet case, Nucl. Phys. B691, 129 (2004).

[59] A. Falkowski and J. F. Kamenik, Diphoton portal to warped gravity, Phys. Rev. D 94, 015008 (2016). 